\title{
ARBEITSGRUPPE VERKEHRSMITTEL
}

\author{
(Helmut Eichlseder, Eberhard Schutting, 17.09.2019)
}

\section{EINLEITUNG}

Das umfangreiche Gebiet der Verkehrsmittel wird in den folgenden Ausführungen auf die repräsentativen Verkehrsmittel Personenkraftfahrzeuge (Pkw), Leichte Nutzfahrzeuge (LNFZ), Lastkraftwagen (LKW) und Omnibusse, motorisierte Zweiräder sowie schienengebundene Fahrzeuge bezogen fokussiert, eine vollständige Darstellung ist naturgemäß in dem zur Verfügung stehenden Umfang nicht möglich. Bezug wird vorwiegend auf die europäischen gesetzlichen Rahmenbedingungen genommen.

Als besonders relevante Herausforderungen in der Gruppe „Verkehrsmittel“ wurden zwei Punkte gesehen, nämlich 1.) die Schadstoffemissionen und 2.) der Einsatz von Primärenergie und wertvollen Rohstoffen über den gesamten Lebenszyklus des Verkehrsmittels (Herstellung, Betrieb, Entsorgung) und die damit verbundene Treibhausgasrelevanz, insbesondere den $\mathrm{CO}_{2}$ Ausstoß. Daher wird im Folgenden auf diese beiden Punkte fokussiert.

In der Vorschau sollten sich die Aussagen auf einen Zeitraum bis etwa 2030 beziehen und einen möglichen Ausblick auf 2050 geben.

\section{DANKSAGUNG}

Der Dank der Autoren gilt Herrn Professor Peter Veit für die Berichtsteile zum Thema des nachhaltigen Schienenverkehrs und Herrn Professor Andreas Kugi für die kritische Durchsicht des Gesamtberichtes.

\section{SCHADSTOFFEMISSIONEN}

Die Abgase beinhalten als limitierte Schadstoffe Stickoxide $\left(\mathrm{NO}_{\mathrm{x}}\right)$, Kohlenstoffmonoxid (CO) und Kohlenwasserstoffe (HC) sowie Partikelmasse und zukünftig Partikelanzahl (PM und PN). Bei Nutzfahrzeugen werden ab EURO VI zusätzlich $\mathrm{NH}_{3}$ und $\mathrm{CH}_{4}$ (bisher nur für Erdgasfahrzeuge) limitiert.

Es gibt eine Reihe von Normen, die die Grenzwerte für $\mathrm{CO}, \mathrm{NO}_{\mathrm{x}}, \mathrm{HC}$ und PM je nach Art des Motors (Otto- oder Dieselmotor) und nach Kraftfahrzeugtyp (Pkw, LNFZ, LKW und Omnibusse, Zweiräder oder schienengebundene Fahrzeuge) festlegen. In den letzten Jahrzehnten wurden in allen industrialisierten Ländern gesetzliche Bestimmungen zur Begrenzung der Abgasemission der oben angeführten Fahrzeuge erlassen. Die erlaubten Grenzwerte wurden seit Beginn der Regelung kontinuierlich abgesenkt und die zugehörigen Prüfverfahren wurden mehrmals mit dem Ziel, die Entwicklung der Technik sowie die beim realen Betrieb des Fahrzeuges auftretenden Randbedingungen zu berücksichtigen, modifiziert. Während die Messverfahren heute in wesentlichen Teilen weltweit einheitlich sind, bestehen hinsichtlich der Prüfzyklen und der Grenzwerte zum Teil noch erhebliche Unterschiede. Die bestehenden Unterschiede wirken sich in vielen Fällen sehr stark auf die technischen Lösungen aus, die zur Erfüllung der gesetzlichen Bestimmungen zur Anwendung kommen. 
Als Basis für die Bestimmung der Abgasemissionen bei Pkw und bei Zweirädern wird ein auf einem temperaturkonditionierten Rollenprüfstand zu absolvierender Prüfzyklus herangezogen. Dieser Prüfzyklus ist ein vorgegebener zeitlicher Verlauf der Fahrgeschwindigkeit über eine definierte Wegstrecke, bei dem die Masse der emittierten Schadstoffe bestimmt und dann auf die zurückgelegte Wegstrecke bezogen wird. Das Fahrzeug wird dabei mit der angetriebenen Achse auf einen Rollensatz gestellt, der entsprechend dem Fahrwiderstand (Roll-, Beschleunigungs- und Luftwiderstand) gebremst wird. Seit Herbst 2017 wird dafür der sogenannte WLTC (World Harmonized Test Cycle) herangezogen, der den bis dahin gültigen NEDC ersetzt [1]. Der NEDC wurde seit längerer Zeit dafür kritisiert veraltet zu sein und moderne Fahrprofile nicht mehr ausreichend zu repräsentieren. Aus Gründen der Konsistenz blieb der NEDC aber für die gesetzliche Ermittlung der $\mathrm{CO}_{2}$-Emissionen zunächst gültig, d.h. er dient weiterhin zur Ermittlung der Flottenverbrauchsgrenzwerte $\left(95 \mathrm{~g} / \mathrm{km} \mathrm{CO}_{2}\right.$ ab 2020). Der WLTC wird vorerst nur in Europa verwendet.

Abbildung 1 zeigt die Entwicklung von Grenzwerten am Beispiel der europäischen Gesetzgebung für Diesel-Pkw im Laufe der letzten 30 Jahre und im Vergleich die strengste kalifornische Emissionsstufe SULEV (ㅁuper UItra Low Emission Vehicle).

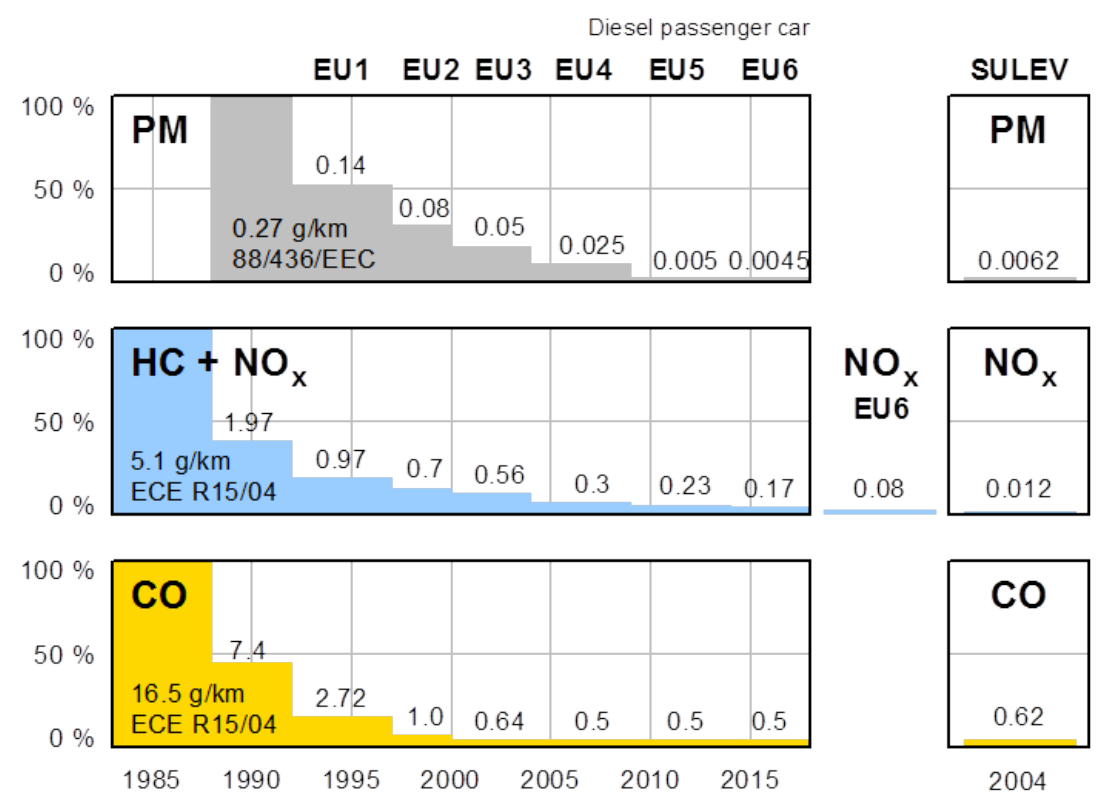

ABBILDUNG 1: ENTWICKLUNG EMISSIONSSTANDARDS FÜR DIESEL PKW IN EUROPA.

Ähnlich ausgeprägt waren die Minderungen der Grenzwerte für Pkw Ottomotoren und LKW. Gegenüber den darin festgelegten drastischen Minderungsstufen ist eine deutliche Verzögerung von etwa 10 bis 15 Jahren bei den entsprechenden Grenzwertstufen für Mopeds und Motorrädern festzustellen, die angesichts der vergleichsweise geringen km-Leistung und der einfachen und kostengünstigen Technik in den früheren Phasen der Emissionslimitierung zunächst auch angemessen waren - Abbildung 2. Mit der nun erfolgten Senkung des Emissionsniveaus von Pkw und LKW tragen aber zum Beispiel die HC Emissionen von Zweirädern trotz des geringen Anteiles an der Transportleistung mittlerweile über $30 \%$ (mit weiter steigender Tendenz) zur verkehrsbedingten HC Emission in Europa bei [2]. Dem wird zukünftig mit einer angepassten Gesetzgebung Rechnung getragen, wobei der endgültige Rahmen derzeit noch nicht fixiert ist. Es ist aber davon auszugehen, dass die Grenzwerte an das Niveau der Pkw-Gesetzgebung angelehnt werden, und dass auch On Board Diagnose verpflichtend wird. In vielen asiatischen Ländern, wo Zweiräder das Straßenbild dominieren, kann dieser Anteil an HC Emissionen bis zu 80 \% betragen und führt damit in diesen Gebieten auch zu massiven Luftgüteproblemen bei HC. 


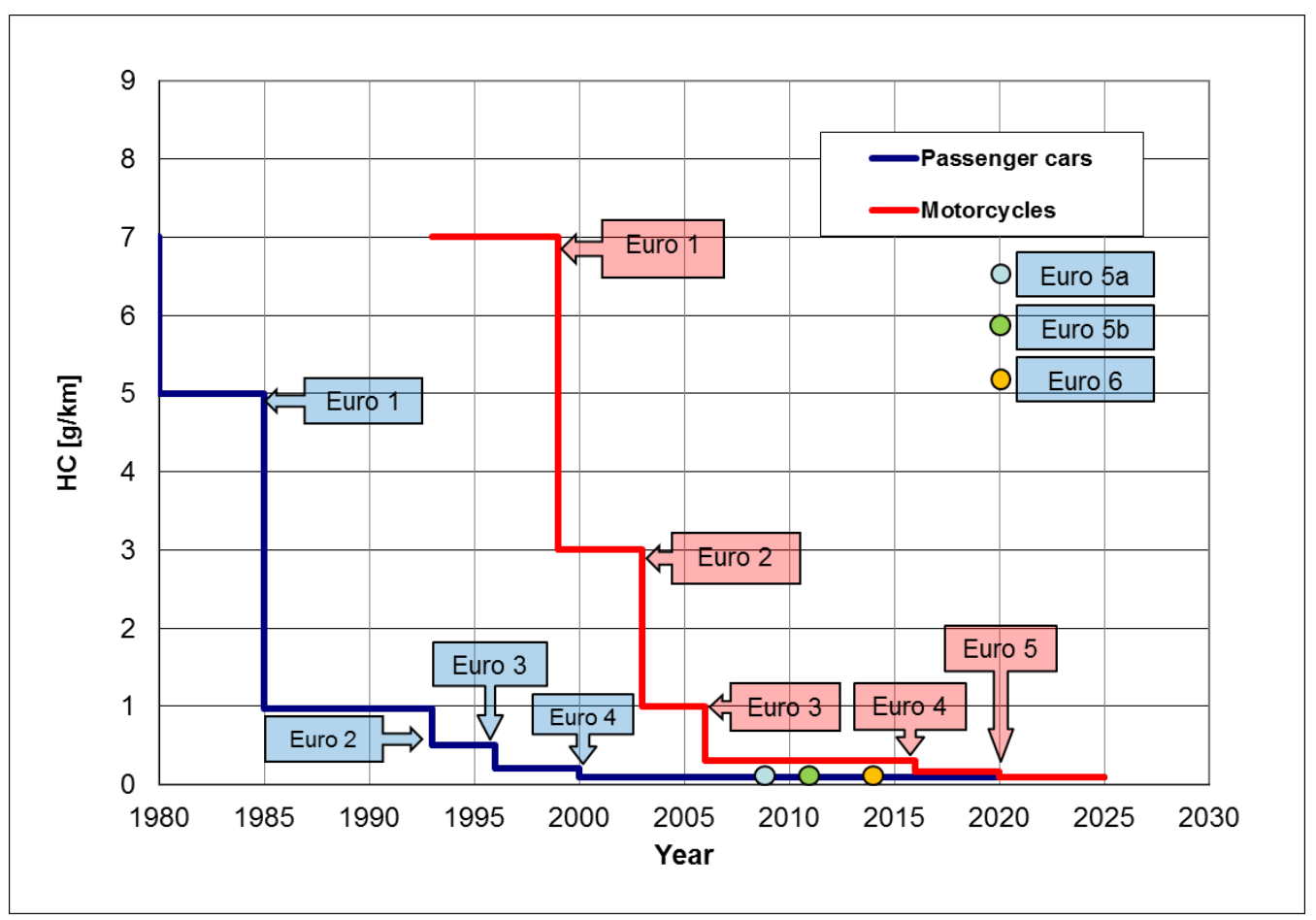

ABBILDUNG 2: ENTWICKLUNG HC EMISSIONSSTANDARDS FÜR ZWEIRÄDER IM VERGLEICH ZU PKW IN EUROPA.

Die auf standardisierte (und weltweit noch nicht einheitliche) Testprozeduren mit definierten Rahmenbedingungen bezogenen Grenzwerte für Fahrzeugemissionen können naturgemäß immer nur einen Teil der möglichen Betriebsbedingungen (Umgebungszustand, Fahrweise, Last- und Drehzahlkollektiv,...) abdecken. Daher wird darüber hinaus in Europa bereits seit Ende 2013 bei LKW, seit Herbst 2017 (mit Euro 6d TEMP) auch bei Pkw, das Emissionsverhalten im realen Fahrbetrieb (RDE Real Drive Emission) bewertet und begrenzt [3]. Mit der Einführung der RDE Regelung hat der Gesetzgeber auf die anhaltenden Vorwürfe reagiert, dass die unter Laborbedingungen gemessenen Emissionierungszyklen (NEDC, WLTC) den realen Fahrbetrieb bei weiten nicht repräsentieren. Diese Debatte wurde durch den Mitte 2015 bekannt gewordenen „VW-Dieselskandal“ deutlich angefeuert. Dadurch rückte auch die Thematik der Emissionsgesetzgebung in das öffentliche Licht (mit allen Vorund Nachteilen einer öffentlichen Diskussion). Der Abbildung 3 ist ein Überblick über die Einführung der verschiedenen gesetzlichen Maßnahmen der Pkw-relevanten Euro 6 Gesetzgebung zu entnehmen [4]. 

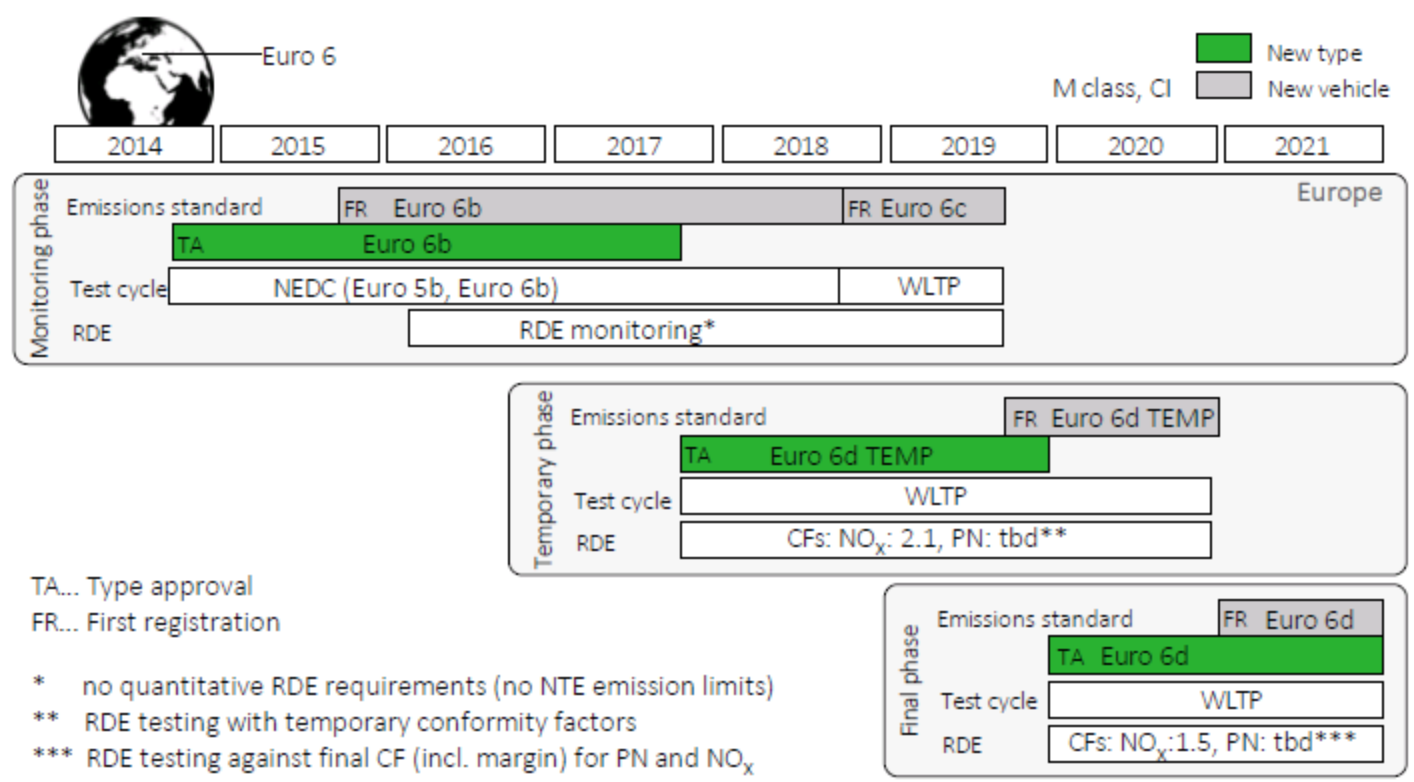

ABBILDUNG 3 ZEITSCHIENE FÜR DIE PKW-EMISSIONSGESETZGEBUNG EURO 6 [4]

Trotz aller in der Vergangenheit erreichten und in Normzyklen nachgewiesenen Verbesserungen bestehen allerdings mit der heute auf der Straße befindlichen Fahrzeugflotte lokal in verkehrsintensiven Gebieten Probleme in der Einhaltung von derzeit definierten Luftgütezielen bezüglich $\mathrm{NO}_{2}$ und Partikel. Diesen haben in Deutschland bereits zu lokalen innerstädtischen Sperren für ältere Diesel-Pkw geführt.

Fokussiert werden in weiterer Folge die Themen Stickoxide $\left(\mathrm{NO}_{\mathrm{x}}\right)$ und Partikel (PM) diskutiert, da bei HC- und CO-Emissionen mit der katalytischen Abgasnachbehandlung, die bei modernen Pkw und LKW den Stand der Technik darstellt, in den letzten 20 Jahren drastische Emissionssenkungen um 90 bis $98 \%$ realisiert werden konnten. Bei den HC- und CO-Emissionen sind heute nur Fahrzeug- und Motorenkategorien auffallend, die über keine wirksame Abgasnachbehandlung verfügen. Dazu gehören beispielsweise kleine Zweiräder (siehe oben), handgehaltene Arbeitsgeräte und stationäre Aggregate. Für diese ist die Gesetzgebung im Allgemeinen, aber auch bezüglich der Grenzwerte, etwa 15 Jahre hinter derjenigen von Pkw und LKW.

Am Beispiel der von Hausberger [5] ermittelten Abgasemissionen verschiedener Verkehrsmittel in Österreich für die Flotten im Jahr 2014 und 2025 kann die auf Personenkilometer bezogene Emission sowohl für den Personen(Abbildung 4) als auch Güterverkehr (Abbildung 5) vergleichend dargestellt werden.

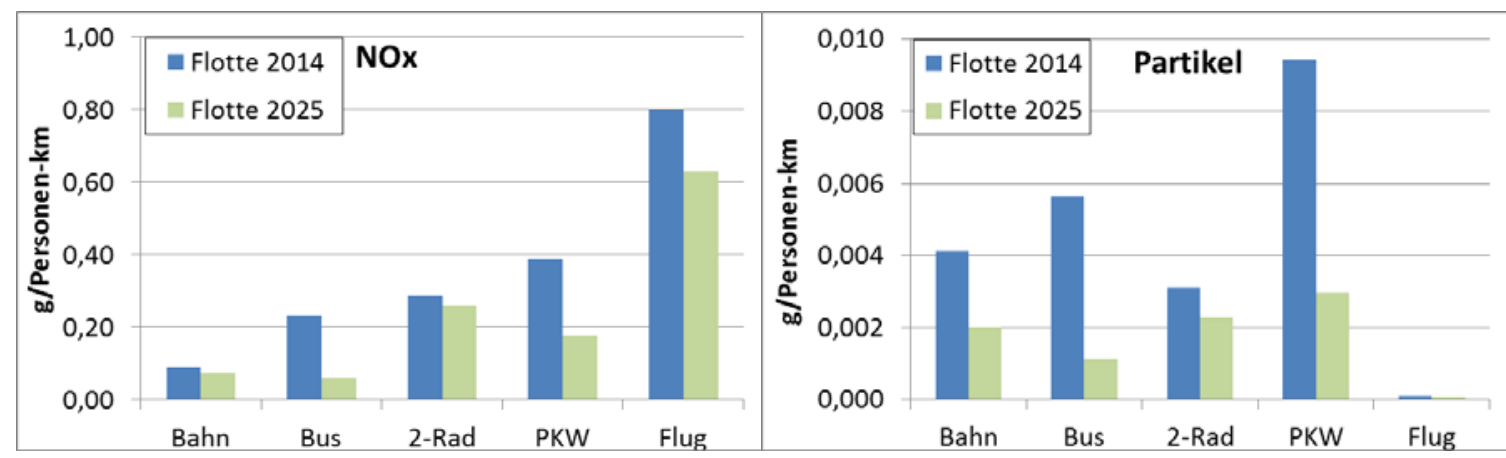

ABBILDUNG 4: PERSONENVERKEHR IN ÖSTERREICH [5]. 


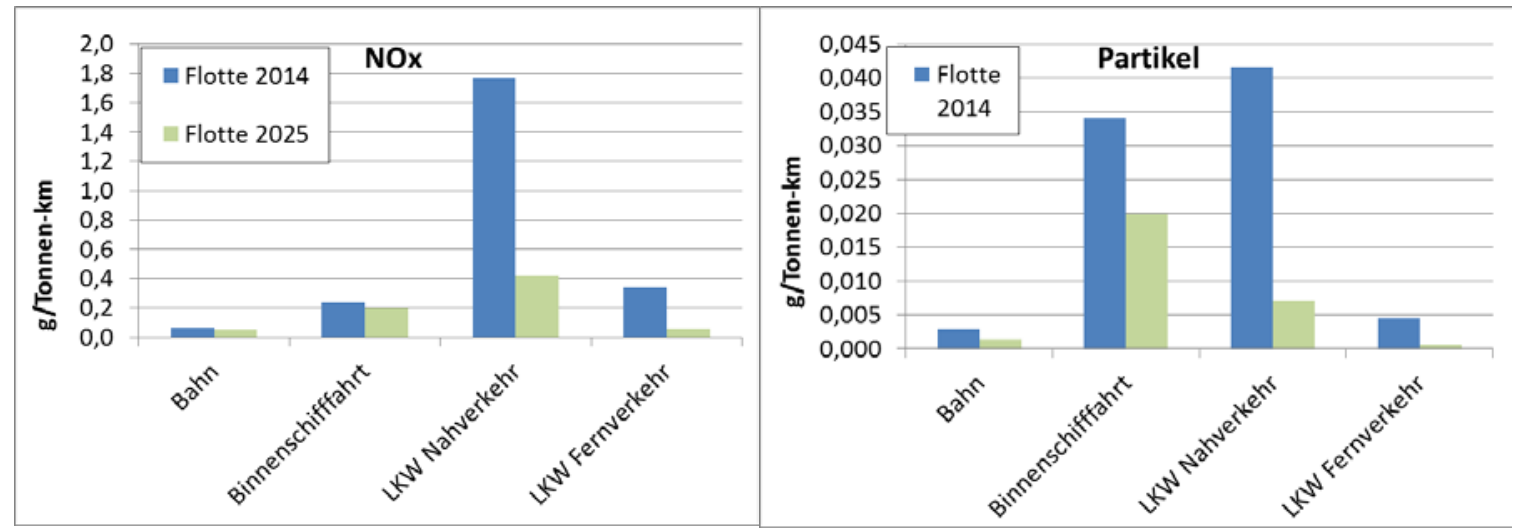

ABBILDUNG 5: GÜTERVERKEHR IN ÖSTERREICH [5].

\section{Feinstaub (Particulate Matter PM):}

Ein Standard zur Kategorisierung der Feinstaubbelastung ist $\mathrm{PM}_{10}$, bei dem jene Partikel in der Atmosphäre berücksichtigt werden, deren aerodynamischer Durchmesser weniger als 10 Mikrometer beträgt. Von der TU Graz [6] wurden diesbezüglich im Jahr 2010 Studien durchgeführt, die die Schadstoffemissionen in österreichischen Städten wie Wien und Linz hinsichtlich der Quellenzuordnung genauer untersuchten. Als Ergebnis konnte festgestellt werden, dass der Verkehr direkt durch Emissionen der Motoren selbst nur einen relativ geringen Anteil von unter $10 \%$ an der gesamten Feinstaubbelastung hat (siehe auch [7]). Der Verkehr kann aber insbesondere in Städten bei bestimmten Wetterlagen indirekt zu einer erhöhten Feinstaubbelastung führen, im Speziellen durch Bremsstaub, Reifenabrieb und vor allem durch Aufwirbelung des bereits vorhandenen Feinstaubs, der neben natürlichen Quellen anthropogene Anteile aus Industrie und Gewerbe hat. In Abb. 4 ist die Entwicklung und Prognose der PM $_{10}$-Emissionen in Wien beispielhaft dargestellt.

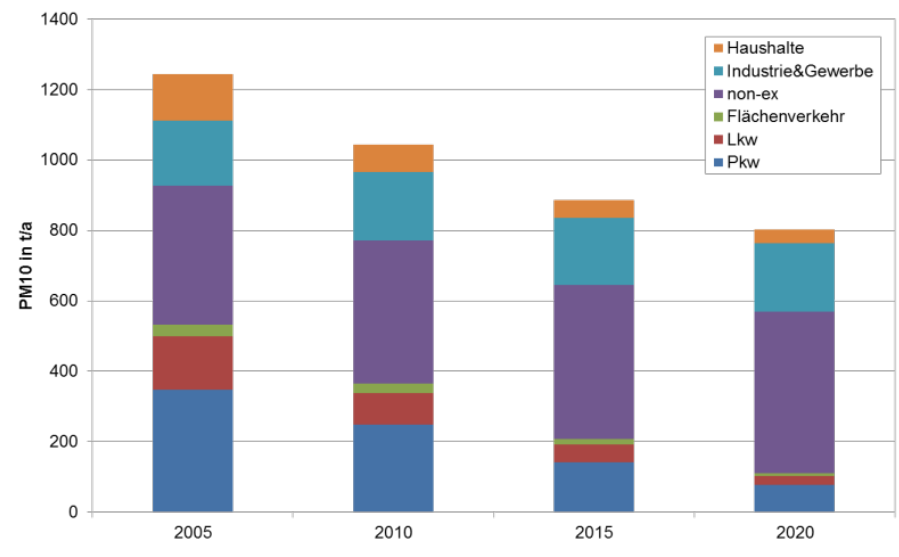

ABBILDUNG 6: EMISSIONEN PM10 IN WIEN [6].

Der Grund für den relativ geringen und deutlich sinkenden Anteil der Motor-Partikel ist sicherlich im Einsatz von effizienten Partikelfiltern in neuen Dieselfahrzeugen, die zunehmenden Anteil in der Fahrzeugflotte haben, zu sehen. Für Neuzulassungen schreibt die Euro 6 Norm seit September 2014 nicht nur Partikel-Massengrenzwerte (von 4,5 mg Partikel/km), sondern seit 2017 auch die gleichen Partikel-Anzahlgrenzwerte für Otto- und DieselPkw (PN < 6×10 11 je km) vor. Auch für Nutzfahrzeuge ist seit EURO VI neben dem Partikel-Massengrenzwert ein Anzahl-Grenzwert eingeführt. Damit soll der gesundheitlichen Relevanz der Partikelanzahl anstelle der Masse Rechnung getragen werden. Da Partikelfilter nicht nur für die Partikelmasse, sondern auch für die Partikelanzahl eine hohe Reduktionswirkung aufweisen, werden diese Anzahl-Grenzwerte von Diesel-Pkw bereits heute unterschritten. Die Regulierung der Partikelanzahl führte dazu, dass seit 2018 auch direkteinspritzende OttoMotoren weitestgehend mit Otto-Partikelfiltern (OPF) ausgestattet werden (während die Partikelmasse für Ottomotoren generell keine Herausforderung darstellt) [8], [9]. 
Insgesamt kann also festgestellt werden, dass mit der verfügbaren Technologie und deren Durchdringung am Markt die motorseitig verursachten Partikel-Masse- und Partikel-Anzahlemissionen auf ein geringes Niveau gebracht werden können.

Betrachtet man die Partikelemission $\mathrm{PM}_{2.5}$, so bleiben als Verursacher in erster Linie verbrennungsbedingte Partikel (Verkehr und Energieerzeugung) sowie sekundär gebildete Aerosole übrig. Derzeit wird intensiv diskutiert und die Forderung erhoben, die heute nur auf der Bezugsgröße $\mathrm{PM}_{10}$ und $\mathrm{PM}_{2.5}$ basierenden Immissionswerte um kleinere Partikel wie die $\mathrm{PM}_{1.0}$ (alveolengängiger Feinstaub) und $\mathrm{PM}_{0.1}$ zu erweitern und diese explizit in die Überlegungen mit einzubeziehen. Hier bestehen allerdings noch offene messtechnische Fragestellungen. Einheitliche Sicht besteht darüber, dass die Gefährlichkeit bzw. Toxizität mit kleineren Partikeln zunimmt und kritischer zu bewerten ist, die Streubreite der Aussagen ist jedoch beträchtlich. Hier besteht weiterer Forschungsbedarf.

\section{STICKOXIDE $\left(\mathrm{NO}_{\mathrm{x}}\right)$ :}

Der Straßenverkehr ist einer der Hauptverursacher bei den Emissionen von Stickoxiden, insbesondere durch Dieselmotoren. Auswertungen der TU Graz [6] zeigen, dass im Jahr 2010 in Wien 60\%, in Linz 35 \% und in Salzburg $50 \%$ der $\mathrm{NO}_{x}$ Emissionen dem Straßenverkehr geschuldet sind. Am Beispiel der weithin bekannten Messstelle Neckartor in Stuttgart ist zu erkennen, dass über die Hälfte der $\mathrm{NO}_{2}$ Emissionen von Fahrzeugen verursacht werden - Abbildung 7 [7].

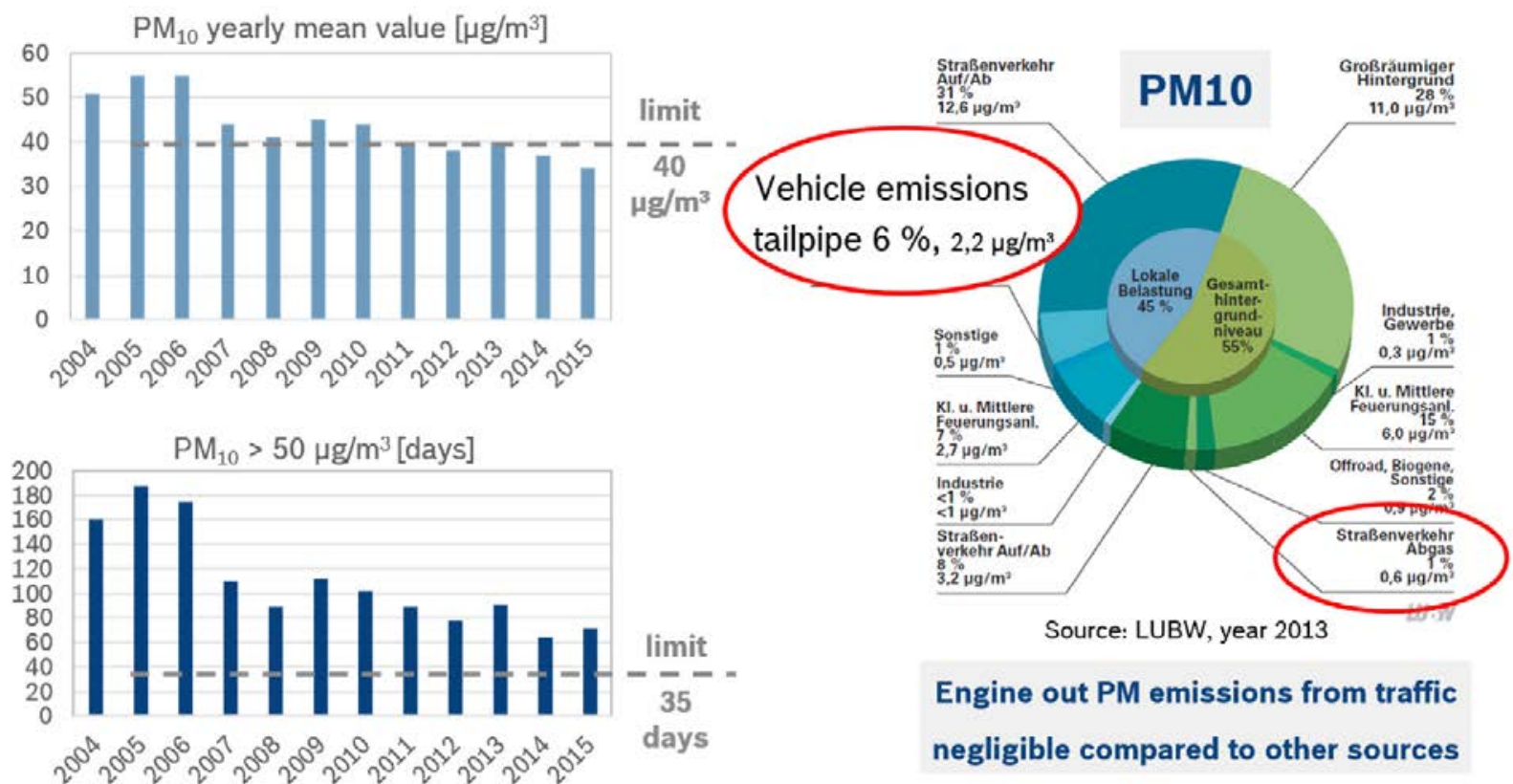

\section{ABBILDUNG $7 \mathrm{NO}_{2}$ - IMMISSIONEN, BEISPIEL STUTTGART NECKARTOR [7]}

So werden auch an einigen verkehrsexponierten Punkten in Österreich die Luftgütegrenzwerte für $\mathrm{NO}_{2}$ häufig überschritten, und eine wesentliche Minderung der verkehrsbedingten $\mathrm{NO}_{2}$-Emissionen analog zu der bei anderen Schadstoffkomponenten konnte in den letzten Jahren nicht festgestellt werden. Gründe dafür sind unter anderem, dass die Limitierung der Fahrzeug-Emissionsgrenzwerte auf $\mathrm{NO}_{\mathrm{x}}$ (also die Summe aus $\mathrm{NO}$ und $\mathrm{NO}_{2}$ ) erfolgt und hier mit modernen Brenn- und Abgasnachbehandlungs-verfahren eine Verschiebung der Anteile in Richtung $\mathrm{NO}_{2}$ erfolgt, sowie der bisherige Testzyklus, der das Realverhalten nicht ausreichend abbildet. Auch wenn die Minderung der $\mathrm{NO}_{2}$-Emissionen unerwartet gering ausfällt, ist nichts desto trotz eine sinkende Tendenz feststellbar, was letztlich auf die verschärften Grenzwerte zurückzuführen ist.

Bereits die Einführung der Euro 6 Norm (2014) mit einem $\mathrm{NO}_{x}$-Grenzwert von $80 \mathrm{mg} / \mathrm{km}$ führte dazu, dass praktisch alle Diesel-Pkw mit einer Stickoxidnachbehandlung ausgestattet wurden, spätestens die RDE Gesetzgebung (2017) erzwang den flächendeckenden Einsatz. Dabei zeichnet sich ab, dass sich - zumindest in 
Europa - die aufwändigeren SCR-Systeme (mit AdBlue - Eindosierung) durchsetzen werden, da die weniger komplexen Speicherkat-Systeme im herausfordernden RDE-Betrieb allein die nötigen Konvertierungsraten nicht erreichen [4].

Bei den LKW sind seit dem Inkrafttreten der EURO VI Stufe mit einem Grenzwert von 0,4 g/kWh alle Fahrzeuge mit einem SCR-System ausgestattet.

Die für Euro 6 erforderliche Abgasnachbehandlung wird in Verbindung mit der Bewertung und Limitierung der Real Drive Emissionen bei einer in einigen Jahren erreichten Flottendurchdringung einen wesentlichen Beitrag zur Emissionssenkung liefern, welche auch die Überschreitung der $\mathrm{NO}_{2}$ Luftgütewerte vermeiden sollte. Diese Einschätzung kommt auch in dem Statement

„Unseres Erachtens wird die angelaufene RDE-Regulierung durch die breite Abdeckung der möglichen Fahr-, Topografie- und Umweltrandbedingungen die Immissionssituation speziell an den kritischen Hotspots deutlich verbessern." [10]

zum Ausdruck, welches sinngemäß die Einschätzung vieler Experten wiedergibt. Auch erste Messungen bescheinigen der RDE-Gesetzgebung die erwünschte Wirkung auf die Realemissionen von Pkw - siehe Abbildung 8 [11]. Dargestellt sind die durchschnittlichen $\mathrm{NO}_{x}$-Emissionen mehrerer Pkw, getrennt nach Schadstoffnorm. Die Messungen wurden nach dem Verfahren des ADAC-Ecotest [12] durchgeführt ${ }^{1}$. Der Durchschnitt der EU6d-Temp Kategorie wurde dabei aus 25 Fahrzeugen unterschiedlichster Hersteller gemittelt. Den anderen Kategorien liegen 69 bzw. 149 Fahrzeuge zugrunde.

\section{NO $_{x}$-Emissionen im Ecotest nach Schadstoffnorm}

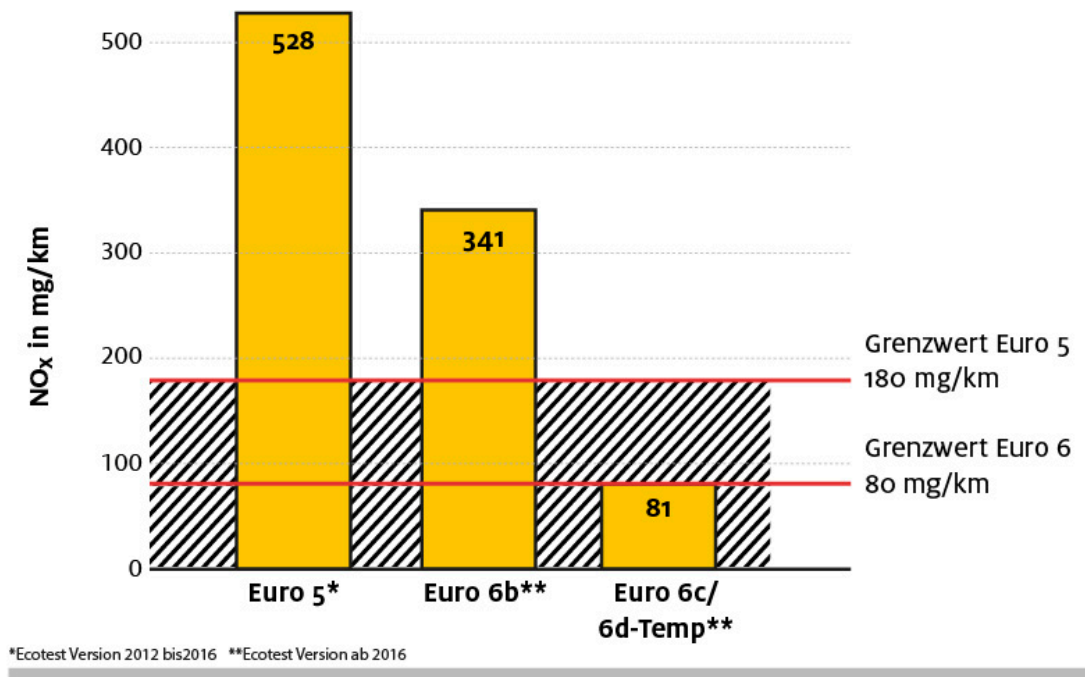

Q 9.2018ADAC e.V.

ABBILDUNG 8 NOX-EMISSIONEN IM ADAC-ECOTEST NACH SCHADSTOFFNORM [11]

Im Verlauf des letzten Jahres hat sich zusätzlich eine Diskussion über die Sinnhaftigkeit der Immissionsgrenzwerte entwickelt. Ein Standpunkt in dieser Diskussion ist, dass die Grenzwerte willkürlich und unsinnig tief angesetzt wurden. So wird zum Beispiel argumentiert, dass die erlaubte Konzentration von $\mathrm{NO}_{\mathrm{x}}$ am Arbeitsplatz 20 mal höher ist, als der Grenzwert der Immissionsgesetzgebung. Die Vorgehensweise bei der Ermittlung der Grenzwerte wurde zuletzt von einer Gruppe deutscher Lungenärzte stark kritisiert [13].

\footnotetext{
${ }^{1}$ Die Messungen wurden bis Euro 5 nach der Ecotest-Version 2012 bis $2016\left(^{*}\right)$ [56] durchgeführt, ab Euro 6b nach der Version ab 2016 [57] (**)
} 
"Es ist sehr wahrscheinlich, dass die wissenschaftlichen Daten, die zu diesen scheinbar hohen Todeszahlen führen, einen systematischen Fehler enthalten. Eine genauere Analyse der Daten zeigt, dass diese extrem einseitig interpretiert wurden, immer mit der Zielvorstellung, dass Feinstaub und $\mathrm{NO}_{x}$ schädlich sein müssen. Andere Interpretationen der Daten sind aber möglich, wenn nicht viel wahrscheinlicher. " [13]

\section{KOHLENDIOXID $\mathrm{CO}_{2}$}

$\mathrm{CO}_{2}$ spiegelt als Produkt der vollständigen Verbrennung den Kraftstoffverbrauch wieder. Es ist nicht unmittelbar gesundheitsschädlich, ist aber ein Treibhausgas und damit klimaschädlich. Es muss somit von den gesundheitsschädlichen echten Schadstoffen NOx, $\mathrm{HC}, \mathrm{CO}$ und Partikel unterschieden werden. Die Menge an $\mathrm{CO}_{2}$ Emissionen ist um ein Vielfaches über der der Schadstoffemissionen.

Anthropogenes $\mathrm{CO}_{2}$ entsteht in vielen Quellen. Um den Gesamtausstoß zu verringern wurden durch die EU auch für den Verkehrssektor Grenzwerte eingeführt. 2021 dürfen die verkauften Pkw im Flottenverbrauch im Mittel höchstens $95 \mathrm{gCO}_{2}$ pro Kilometer ausstoßen. Im Dezember 2018 wurde eine Verschärfung dieses Wertes um 37,5 \% bis 2030 beschlossen. Dieser Grenzwert stellt eine außerordentliche Herausforderung für die Fahrzeughersteller dar. Die Regulierung setzt keinen europäischen Einheitswert, sondern berücksichtigt grundsätzlich die unterschiedlichen Produktpaletten der Konzerne. So muss nicht jeder einzelne Hersteller den europäischen Gesamtflottenwert von $95 \mathrm{~g} / \mathrm{km}$ bei Pkw einhalten. Vielmehr wird für jeden Hersteller ein spezifischer Grenzwert errechnet, der auf dem durchschnittlichen Fahrzeuggewicht der Herstellerflotten beruht. Im Durchschnitt aller Hersteller ist damit statistisch sichergestellt, dass der europäische Flottenwert erreicht wird [14].

\section{$\mathrm{CO}_{2}$-Ausstoß von Neuwagen - EU-Ziel 2030}

Durchschnittliche Emissionen aller Pkw-Neuzulassungen, $\mathrm{g} / \mathrm{km}$

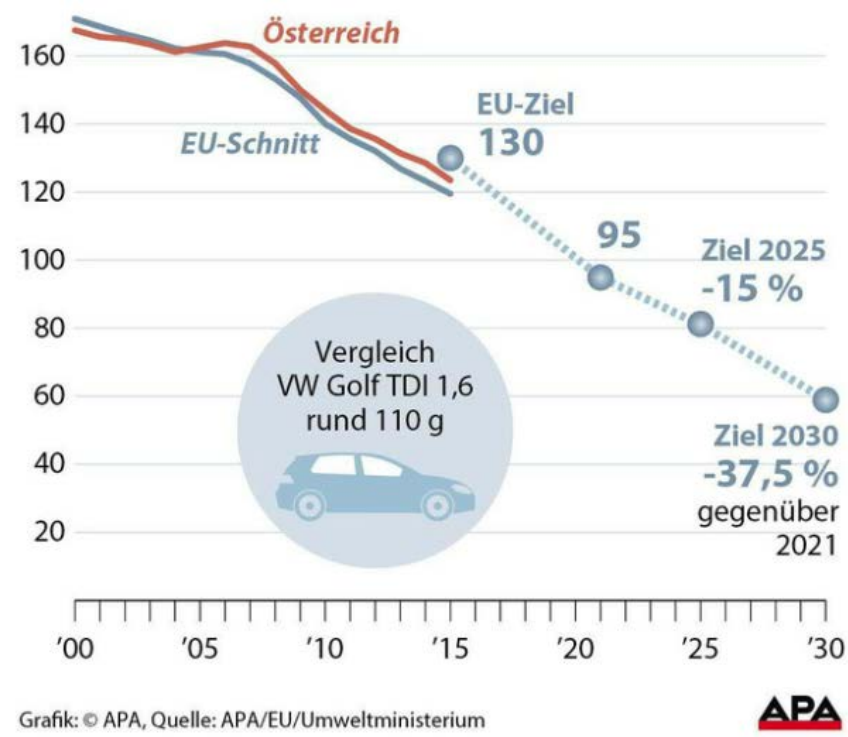

\section{ABBILDUNG $9 \mathrm{CO}_{2}$ GRENZWERTE BIS 2030 FÜR PKW [ [15]]}

Für Nutzfahrzeuge gilt eine ähnliche Verschärfung, hier wurde seitens EU eine stufenweise Verringerung der $\mathrm{CO}_{2}$ Emisisonen auf -30 \% im Jahr 2030 (ausgehend vom Niveau 2019) beschlossen. Zusätzlich wurde mit Beginn 2019 in der EU für die Bestimmung der $\mathrm{CO}_{2}$-Emissionen von schweren Nutzfahrzeugen ein neues Verfahren eingeführt, bei dem jede verfügbare Fahrzeugkonfiguration mithilfe eines standardisierten Simulationswerkzeuges individuell bewertet wird [16]. Dieses Werkzeug - VECTO - verwendet gemessene Kennwerte von verschiedenen Baugruppen (Motorkennfeld, Luftwiderstand der Karosserie, Getriebewirkungsgrad, etc.) eines Schwerfahrzeuges und berechnet daraus die $\mathrm{CO}_{2}$-Emissionen von bestimmten Fahrzeugvarianten. Auch für die Nutzfahrzeughersteller stellt dieser Grenzwert eine außerordentliche Herausforderung dar. 
Verschiedene Maßnahmen zur Erreichung dieser Grenzwerte stehen zur Diskussion. Sie sind für Pkw und Nutzfahrzeuge relativ ähnlich. Die Erhöhung der Effizienz von Motor, Antrieb und Fahrzeug kann vermutlich nur mehr einen beschränkten Beitrag leisten, da das Potential weitestgehend ausgeschöpft scheint. Die Verwendung $\mathrm{CO}_{2}$-neutraler Kraftstoffe erscheint als interessante Lösung, jedoch bestehen noch große Hürden auf dem Weg zur flächendeckenden Umsetzung. Insbesondere ist die Gesetzgebung hier unter Zugzwang, denn momentan würde eine eventuelle $\mathrm{CO}_{2}$-Einsparung durch derartige Kraftstoffe nicht in die $\mathrm{CO}_{2}$-Bilanz der Hersteller eingerechnet [17].

Der Einsatz elektrischer Antriebe und Plug-In Antriebe stellt aus heutiger Sicht die einzig praktikable Lösung zur Erreichung der Ziele der Gesetzgebung dar. Allerdings nur aufgrund einer in der Gesetzgebung unterstellten $\mathrm{CO}_{2}$ Freiheit von elektrischer Energie, was aus Sicht der Kommission "Nachhaltige Mobilität" - und aus technischer Sicht - vollkommen ungerechtfertigt ist (siehe Kapitel "Einsatz von Primärenergie und wertvollen Rohstoffen über den gesamten Lebenszyklus des Verkehrsmittels (Herstellung, Betrieb, Entsorgung) und der damit verbundene $\mathrm{CO}_{2}$ Ausstoß"). Im Nutzfahrzeugbereich bestehen wesentlich höhere Reichweitenanforderungen, die von batterieelektrischen Antrieben nicht praktikabel erfüllt werden können. Aus diesem Grund erscheint dort der Einsatz von Wasserstoff-Brennstoffzellen wahrscheinlicher.

Die Notwendigkeit die von Menschen verursachten $\mathrm{CO}_{2}-$ Emissionen zu reduzieren, steht außer Frage. Indes, der Verkehr ist nicht der einzige Sektor der menschlichen Gesellschaft, der $\mathrm{CO}_{2}$ produziert, obwohl in der öffentlichen Diskussion und Wahrnehmung das oft so erscheint. Die Kommission sieht sich auch in der Pflicht solche verzerrten Wahrnehmungen zurechtzurücken. So ist es zum Beispiel interessant zu wissen, dass nur rund $1 \%-1,5 \%$ der globalen anthropogenen $\mathrm{CO}_{2}$-Emissionen vom europäischen Pkw-Sektor verursacht werden z.B. $[18]^{2}$.

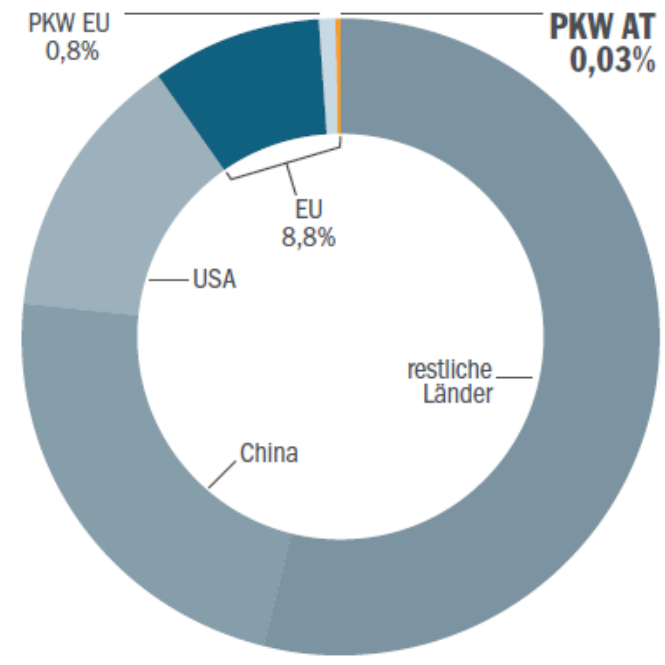

ABBILDUNG 10 ANTEIL DER EUROPÄISCHEN PKW-CO 2 -EMISSIONEN AM GLOBALEN AUSSTOß [18]

\section{EINSATZ VON PRIMÄRENERGIE UND WERTVOLLEN ROHSTOFFEN ÜBER DEN GESAMTEN LEBENSZYKLUS DES VERKEHRSMITTELS (HERSTELLUNG, BETRIEB, ENTSORGUNG) UND DER DAMIT VERBUNDENE $\mathrm{CO}_{2}$ AUSSTOß}

Heutzutage ist die Diskussion über die Umweltfreundlichkeit von Verkehrsmitteln teilweise geprägt von der zum Antrieb notwendigen Energie und dem damit verursachten $\mathrm{CO}_{2}$ Ausstoß pro gefahrenen Kilometer. Als Beispiel

\footnotetext{
2 Je nach Quelle werden etwa 9-12 \% der globalen anthropogenen $\mathrm{CO}_{2}$-Emissionen in der EU ausgestoßen [43], [45], [44]. Davon entfallen in etwa 20 - $25 \%$ auf den Straßenverkehr [46], [47], [48] wovon wiederum 45-60 \% vom Pkw emittiert werden [48], [43].
} 
sind die auf den Personenkilometer bezogenen $\mathrm{CO}_{2}$-Emission verschiedener Verkehrsmittel in Österreich für die Flotten im Jahr 2014 und 2025 sowohl für den Personen- als auch Güterverkehr in Abb. 5 vergleichend dargestellt.

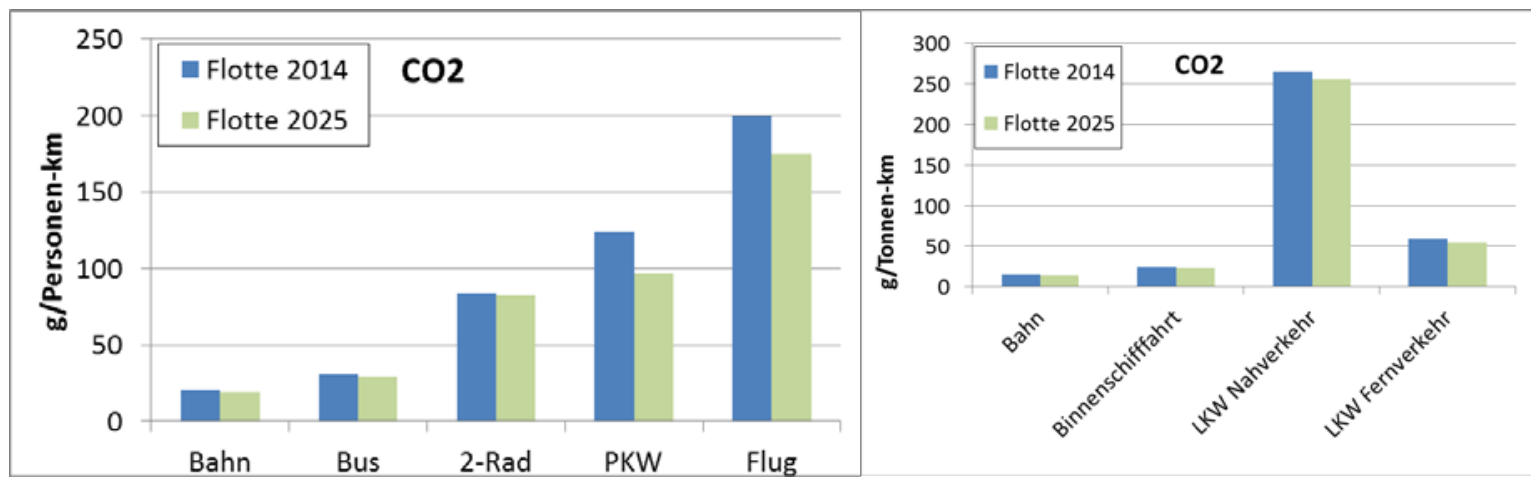

ABBILDUNG 11: $\mathrm{CO}_{2}$-EMISSIONEN DES PERSONEN- UND GÜTERVERKEHRS IN ÖSTERREICH [2].

Die Prognosen über die Art zukünftiger Antriebe fallen teilweise recht unterschiedlich aus, jedoch ist sich die Mehrzahl der Autoren einig, dass es einerseits zu einer großen Vielfalt an nebeneinander existierenden Lösungen kommen wird, und andererseits der Verbrennungsmotor auf längere Sicht - auch im Pkw Bereich - wichtig bleiben wird [19], [20], [21], [22], [23], [24]. Neben den rein verbrennungsmotorischen Antrieben auf Basis von Diesel und Benzin gibt es erdgasbetriebene Fahrzeuge, Hybrid-Antriebe (HEV), Plug-in Hybrid-Antriebe (PHEV), rein elektrische Antriebe (BEV) und Brennstoffzellenantriebe. Abbildung 12 zeigt ein beispielhaftes Szenario für die Entwicklung der Neuzulassungen nach Antriebsarten für Österreich.

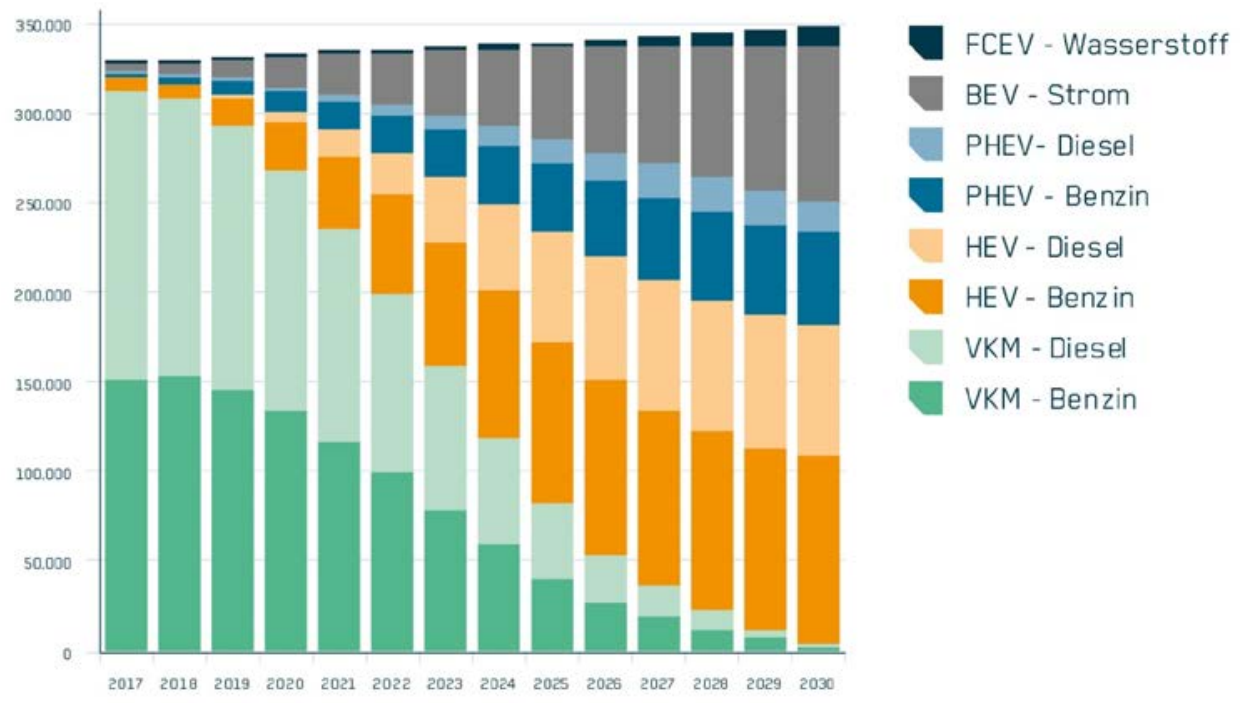

ABBILDUNG 12 ANTRIEBSTECHNOLOGIEAUFTEILUNG PKW - NEUZULASSUNGEN ÖSTERREICH [24]

Insbesondere bei der Bewertung von elektrifizierten Antriebskonzepten wird häufig nur die notwendige elektrische Energie für den Betrieb des Verkehrsmittels herangezogen, ohne den Vorgang der Stromerzeugung mit teilweise sehr niedrigen Wirkungsgraden zu berücksichtigen. Diese eingeschränkte Sichtweise, die sich übrigens auch in den EU Regularien mit per Definition $\mathrm{CO}_{2}$ freiem Strom (!) niederschlägt, birgt die Gefahr, falsche Schlüsse zu ziehen [25].

So kann beispielsweise Norwegen seinen kompletten Strombedarf aus erneuerbarer Energie decken [26], wohingegen in China ca. $80 \%$ des Stromes in Kohlekraftwerken erzeugt wird [27]. Die $\mathrm{CO}_{2}$ Emissionen eines Kohle-Kraftwerks ohne Kraft-Wärmekopplung liegen bei mehr als $1000 \mathrm{~g} / \mathrm{kWh}$ (im Vergleich zu $23 \mathrm{~g} / \mathrm{kWh}$ von Windkraftanlagen, $10 \mathrm{~g} / \mathrm{kWh}$ von Wasserkraftanlagen) [28] und sind damit im Vergleich zu den $\mathrm{CO}_{2}$ Emissionen, 
die bei einem Fahrzeugantrieb mit Verbrennungskraftmaschine je Kilowattstunde auftreten (> $300 \mathrm{~g} / \mathrm{kWh}$ [29]), deutlich höher, auch wenn man für die Förderung, den Transport und die Herstellung der Treibstoffe noch weitere $15-20 \%$ hinzunimmt. Die Umweltfreundlichkeit des Betriebes eines Elektrofahrzeuges hängt also wesentlich von der Art der Stromerzeugung ab.

Die Einbeziehung der gesamten Produktionskette eines Energieträgers (Kraftstoffes) wird als "Well-to-Wheel“Betrachtung bezeichnet. Sie gilt für alle Kraftstoffarten (Strom, Wasserstoff, etc.). Diese Well-to-WheelBetrachtung, also von der Quelle bis zum Rad, jedoch ohne die Infrastruktur hat sich heutzutage in den meisten Vergleichen bereits durchgesetzt [30].

Um einen noch besseren Vergleich der unterschiedlichen Verkehrsmittel (Pkw, LKW und Omnibusse, Zweiräder und Mopeds sowie schienengebundene Fahrzeuge) bzw. der unterschiedlichen Antriebskonzepte (Ottomotor, Dieselmotor, HEV, PHEV, battery electric vehicle (BEV), Brennstoffzelle, Erdgas) anstellen zu können, ist es notwendig, nicht nur den Betrieb, sondern den gesamten Lebenszyklus von der Erzeugung des Verkehrsmittels über den Betrieb bis zur Entsorgung inklusive Recycling sowie die Errichtung und Instandhaltung der dafür notwendigen gesamten Infrastruktur (Straßen, Schienennetze, Stromversorgung, Tankstellennetz etc.) anzusehen. Diese Betrachtung wird im allgemeinen als LCA („Life Cycle Assessment“) bezeichnet, häufig auch als "Cradle-to-Grave"-Betrachtung. Selbstverständlich ist diese Betrachtungsweise vorzuziehen, wenn die globale Auswirkung der Mobilität bewertet werden soll. Jedoch stellt eine LCA eine ungeheure Herausforderung dar, und ist darüber hinaus sehr stark von den letztendlich angenommen Systemgrenzen abhängig.

Nichts desto trotz geben die Literaturstellen ähnliche Werte für die Bewertung unterschiedlicher Antriebsarten an, z.B. [31], [20]. Es besteht ein genereller Konsens darüber, dass Elektroautos beim europäischen Strommix über Lebensdauer bei LCA Betrachtung einen leichten Vorteil bei den $\mathrm{CO}_{2}$ Emissionen erreichen können, während typischerweise China als Negativbeispiel für das Gegenteil angeführt wird. Neben der $\mathrm{CO}_{2}$-Emissionen der Stromerzeugung fällt bei den BEV vor allem auch die Batterieherstellung besonders ins Gewicht. Die dabei entstehenden $\mathrm{CO}_{2}$-Emisisonen führen dazu, dass $\mathrm{BEV}$ gewissermaßen mit einem $\mathrm{CO}_{2}$-Nachteil starten, und erst eine beträchtliche Laufleistung absolvieren müssen, bevor überhaupt ein Gleichstand mit konventionellen Fahrzeugen erreicht ist. Die nötige Laufleistung hängt von verschiedenen Parametern ab, außerdem gibt es durchaus unterschiedliche Ergebnisse der verschiedenen Studien. Eine Größenordnung von 50.000 bis $100.000 \mathrm{~km}$ scheint unter den derzeitigen Randbedingungen plausibel zu sein [32].

Es ist davon auszugehen, dass zumindest in Europa der Anteil des Stroms aus erneuerbaren Quellen in Zukunft steigen wird, was zu einer Verbesserung der Bilanz von Elektrofahrzeugen führt. In [33] ist für die aktuelle Situation ein Gleichstand zwischen Diesel und BEV ausgewiesen, während für 2030 ein Vorteil für elektrische Antriebe von etwa $25 \%$ prognostiziert wird - siehe Abbildung 13. Die Angaben beziehen sich hier auf Fahrzeuge mit einer Reichweite von 100km (die Reichweite fließt durch die Batteriegröße stark in die $\mathrm{CO}_{2}$ Bilanz ein). Auch zu sehen ist, dass eine $\mathrm{CO}_{2}$-Freiheit auch in der Zukunft bei weitem nicht zu erwarten ist. 


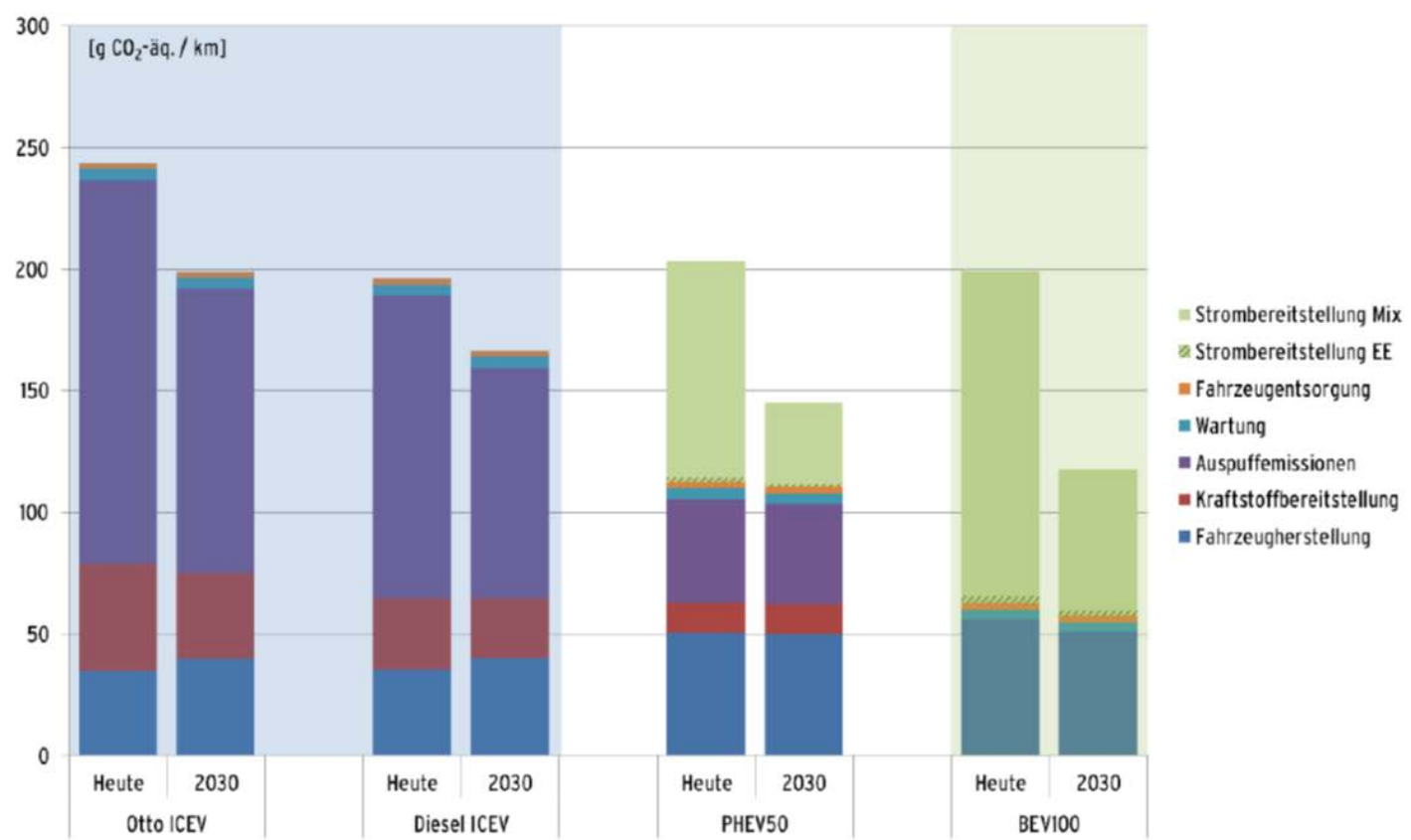

ABBILDUNG 13 CO2-LEBENSZYKLUS-EMISSIONEN; KONVENTIONELLER UND ELEKTROFAHRZEUGE; AUS [34]

Die Wichtigkeit, den gesamten Lebenszyklus eines Fahrzeuges (eines Produktes) in die $\mathrm{CO}_{2}$-Bilanz miteinzubeziehen, soll anhand des Leichtbaus untermauert werden: Leichtbau wird vermutlich ausnahmslos als wirksame und unverfängliche Methode zur Effizienzsteigerung von Pkw angesehen. Durch verringerte Antriebsleistung kann im Fahrbetrieb Kraftstoff gespart werden. Allerdings verbraucht die Herstellung der Leichtbaumaterialien unter Umständen so viel Energie, dass die Bilanz über Lebenszyklus sich sogar verschlechtern kann - siehe Abbildung 14

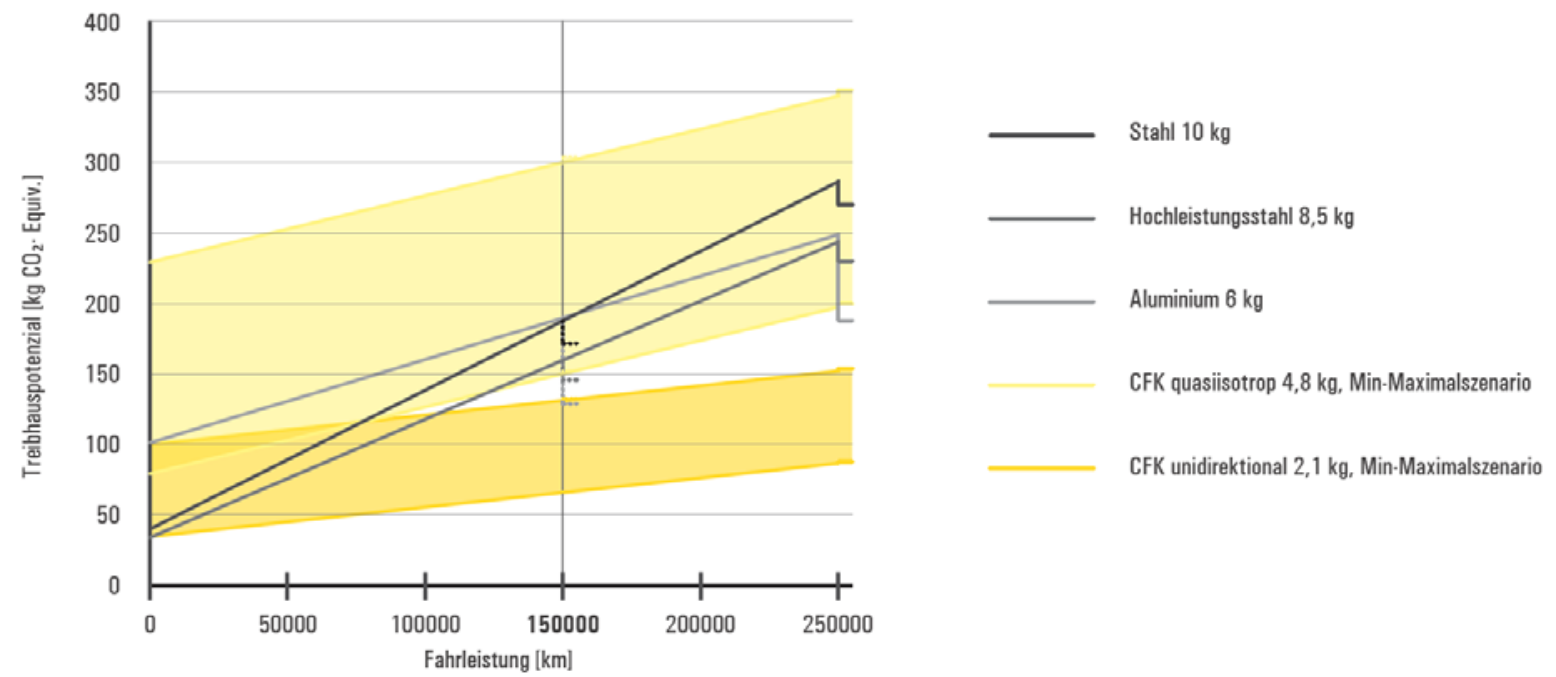

ABBILDUNG 14: TREIBHAUSPOTENZIAL DES LEBENSZYKLUS DER BAUTEILVARIANTEN STAHL, HOCHLEISTUNGSSTAHL UND ALUMINIUM, SOWIE CFK QUASIISOTROP UND UNIDIREKTIONAL (FAHRLEISTUNG: 250.000 KM, BENZINBETRIEBEN) [35]

Der Einsatz von alternativen Leichtbaumaterialien wie Leichtmetalle (Aluminium, Magnesium) oder Faserverbundwerkstoffe (CFK, GFK) verringert das Gewicht des Fahrzeuges und damit den notwendigen Energieeinsatz pro gefahrenen Kilometer (0,3-0,4 I Benzin / 100 kg Fahrzeugmasse). Doch benötigt man für die Herstellung von Leichtbaumaterialien generell mehr Energie als für Stahl.

In einer Studie aus dem Jahr 2012 [35] werden etwa für Aluminium ca. 60,0 kWh/kg, im Vergleich zu 14 kWh/kg bei Stahl, und für CFK sogar 200 kWh/kg angegeben, wobei diese Werte für deutsche Produktionsverhältnisse 
ermittelt wurde. In weiterer Folge wird geschlussfolgert, dass bei einer Laufleistung von 250.000 km Aluminium einen Vorteil bei Pkw erreichen kann, CFK, im Bereich der Bandbreite, aber auch zu einer Verschlechterung führen kann.

Ergänzend sei erwähnt, dass trotz massiver Anstrengungen zur Gewichtsreduktion in den letzten Jahrzehnten das Fahrzeuggewicht zufolge der Sicherheitsanforderungen und der Hinzunahme immer neuer Komfortkomponenten im Wesentlichen gleichblieb.

Die Situation bei Schienenfahrzeugen ist komplett unterschiedlich, hier liegt der Energieanteil der Produktion bei einer Betriebsdauer von 30-40 Jahren unter 5\% und 85\% der eingesetzten Materialien sind bei der Entsorgung stofflich verwertbar. Das Netz der ÖBB weist eine Betriebslänge von 4862 km auf, davon sind 73 \% elektrifiziert, wobei die zweigleisigen Strecken praktisch zur Gänze elektrifiziert sind. Dieselstrecken sind weitgehend nicht im Kernnetz. Daher werden auf den 73 \% elektrifizierten Strecken rund 90\% der Betriebsleistung erbracht.

Die ÖBB decken 2017 rd. 92 \% ihres benötigten Bahnstroms aus Wasserkraft - fast ein Drittel davon wird aus eigenen Kraftwerken gewonnen, ein weiteres Drittel aus Produktion von ÖBB Anlagen in Fremdkraftwerken. Mit einer kontinuierlichen Reduktion der spezifischen $\mathrm{CO}_{2}$-Emissionen von 147.677 tCO2 seit 2006 haben die ÖBB im Jahr 2015 ihre Emissionen gegenüber 2006 um ca. 30\% gesenkt (nach ÖBB Geschäftsbericht 2017). 2018 haben die ÖBB bekanntgegeben, zu 100 \% mit „grünem Strom“ unterwegs zu sein. Die Rekuperation von Bremsenergie ist umgesetzt und erlaubt eine Rückspeisung von rund $25 \%$ der Bremsenergie.

Mit den Energiesparmaßnahmen im ÖBB-Konzern verfolgt die ÖBB das Ziel 7,5 \% des Gesamtenergieverbrauchs (Basisjahr 2014) bis zum Jahr 2020 einzusparen.

Der finanzielle Einsatz, der zur Vermeidung von $\mathrm{CO}_{2}$-Emissionen nötig ist, unterscheidet sich innerhalb der einzelnen Sektoren. Dieser Aspekt spielt in der öffentlichen Diskussion eine untergeordnete Rolle und soll an dieser Stelle explizit erwähnt werden. Eine Studie aus dem Jahr 2012 [33] bescheinigt der Elektromobilität außerordentlich hohe Vermeidungskosten im Vergleich zu Dieselfahrzeugen, zumindest bei einer kurzfristigen Sichtweise bis $2020^{3}$. So werden hier die Kosten eines Umstiegs mit $1500 €$ pro Tonne eingesparten $\mathrm{CO}_{2}$ angegeben - siehe Abbildung 15. Aktuell würde mittels thermischer Sanierung von älteren Gebäuden Kosten von weniger als $150 € / \mathrm{tCO}_{2}$ anfallen. Die gleiche Studie bescheinigt auch, dass langfristig durch die zu erwartende Weiterentwicklung der Technologie im Jahr 2050 der Ersatz von Dieselfahrzeugen durch Elektrofahrzeuge sogar einen Kostenvorteil von -50 bis $-100 € / \mathrm{tCO}_{2}$ entstehen kann - siehe Abbildung 16. Dass die Reduktion der $\mathrm{CO}_{2}$ Emissionen Kosten verursachen wird, steht außer Frage. Diese müssen den Kosten gegenübergestellt werden, die dem Klimawandel zuzuschreiben sind, wie etwa durch Dürren oder Überschwemmungen.

Neben den Kosten ist die Menge an $\mathrm{CO}_{2}$, die vermieden werden könnte, ein wesentlicher Parameter. Dies ist in den Abbildungen dieser Studie als Abszisse aufgetragen. Das größte Potential wird hier dem Ausbau der Windkraftanlagen zugeschrieben. Eingriffe in den Verkehr gelingt demnach kurzfristig praktisch kein Potential, in der langfristigen Perspektive ergibt sich in Summe der Maßnahmen ein moderates Potential.

\footnotetext{
${ }^{3}$ Es ist - zugebenermaßen - widersinnig den aktuellen Stand anhand einer alten Prognose darzulegen. Aufgrund der Durchgängigkeit und der umfassenden Aufbereitung soll dennoch an dieser Quelle festgehalten werden.
} 


\section{$\mathrm{CO}_{2}$-Vermeidung 2020}

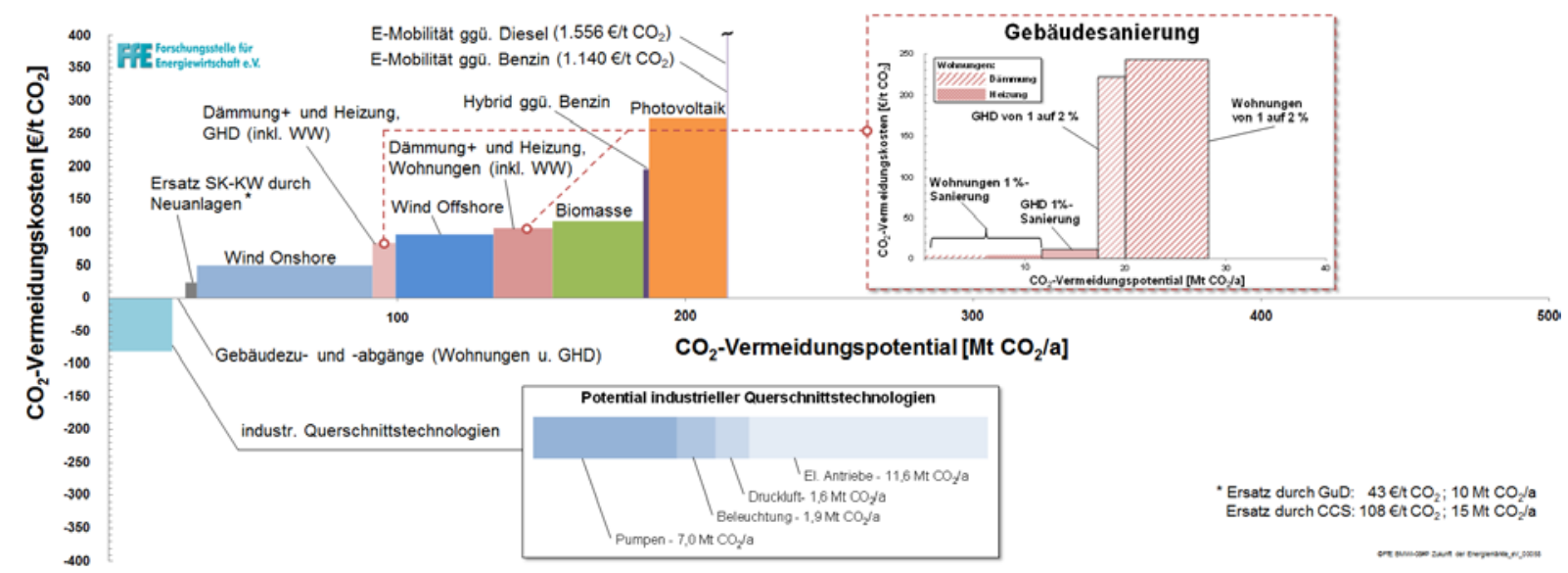

ABBILDUNG $15 \mathrm{CO}_{2}$ VERMEIDUNGSKOSTEN, KURZFRISTIG [33]

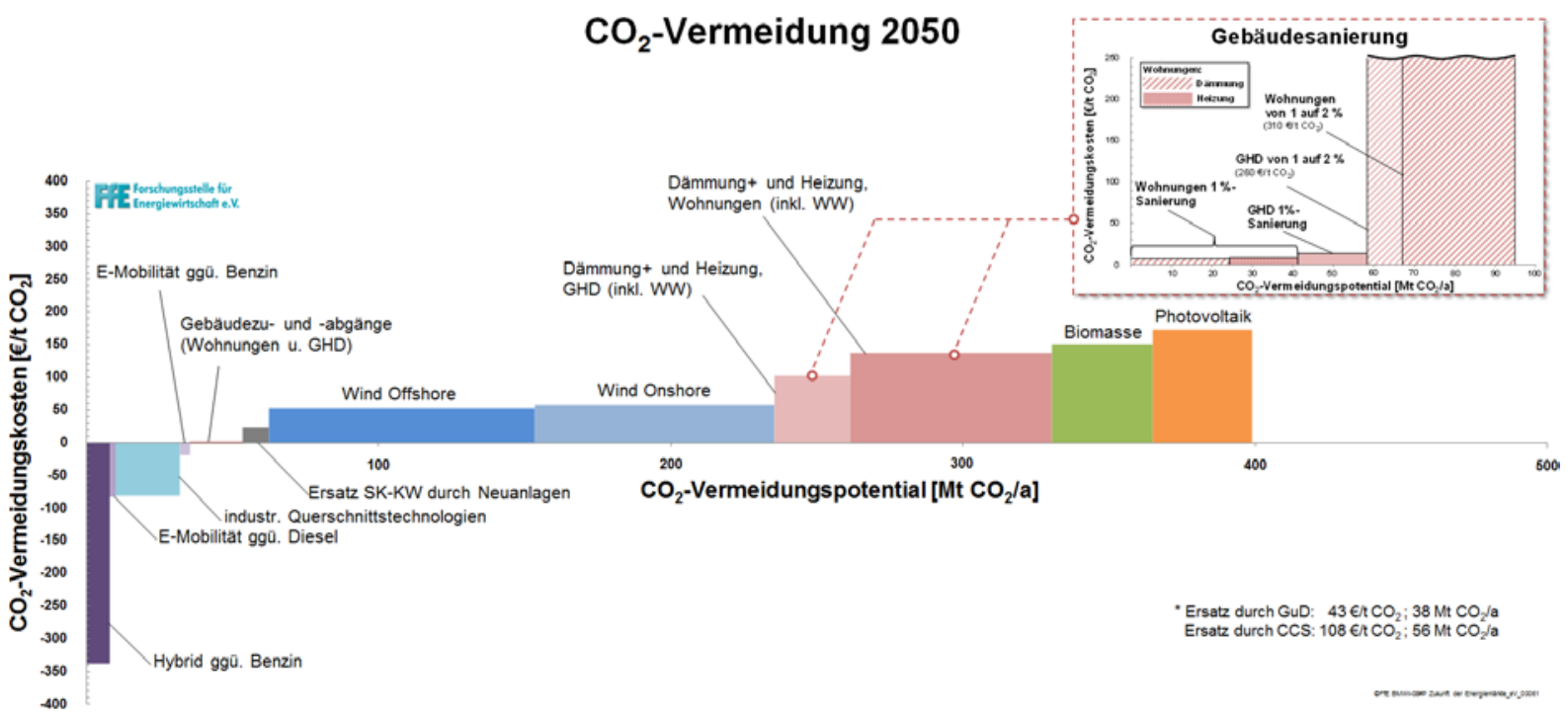

ABBILDUNG $16 \mathrm{CO}_{2}$-VERMEIDUNGSKOSTEN, LANGFRISTIG [33]

Aus dieser Perspektive sollte man bei der Diskussion über $\mathrm{CO}_{2}$ Reduktion auch immer an die weiteren Hauptquellen denken und neben der Optimierung der Verkehrsmittel auch weitere Möglichkeiten in Betracht ziehen.

Bei der Entwicklung der Antriebskonzepte hat sich in den letzten Jahren auch bei Verbrennungskraftmaschinen viel getan, insbesondere konnte der Verbrauch und damit die $\mathrm{CO}_{2}$ Emissionen stetig reduziert werden. Beispielsweise konnte bei Pkw mit Dieselmotoren laut Angabe von BMW von 1998 bis 2018 die Leistung um $40 \%$ und das Drehmoment um 60 \% gesteigert werden bei gleichzeitiger Senkung des Kraftstoffverbrauches um $40 \%$ bei gleichem Fahrzeuggewicht.

$\mathrm{CO}_{2}$-Emissionen entsprechen beim konventionellen Antrieb unmittelbar dem Kraftstoffverbrauch, daher kann auch eine weitere Verbesserung des Wirkungsgrades zur $\mathrm{CO}_{2}$-Einsparung beitragen. Verschiedenste Ansätze bestehen, um den Wirkungsgrad zu steigern. Dazu zählen - motorseitig - Verbesserung des Brennverfahrens durch Variabilitäten, Verbesserung des Aufladesystems, Verringerung der Reibung aber auch zunehmende Elektrifizierung von Nebenaggregaten. Für Motoren mit tendenziell gleichmäßiger Lastanforderung (Fernverkehr) bieten sich Konzepte zur Nutzung der Abwärme wie Turbocompound und nachgeschaltete Wärmekraftprozesse an. Seitens des Antriebs sind in erster Linie Hybrid-Lösungen zielführend, die ja auch schon weit verbreitet sind. Auch bei konventionellen Antrieben können zum Beispiel durch Weiterentwicklung von Getrieben Verbesserungen erzielt werden. 
Jedoch scheinen die Potentiale, die auf diesem Weg zu heben sind, eingeschränkt zu sein. Die Angaben sind vielfältig und weisen oft nur theoretische Werte auf, die einer umfassenden Bewertung nicht standhalten. Mit Sicherheit kann jedenfalls ausgeschlossen werden, dass die $\mathrm{CO}_{2}$-Vorgaben für 2030 bei Beibehaltung der Fahrzeugflotte mittels bloßer Verbesserung konventioneller Antriebe (auch Hybrid) zu bewerkstelligen ist.

Dazu sei folgendes Beispiel angeführt: Ein serienmäßiger 'VW Golf VII 1,6 TDI SCR Comfortline (Diesel)' hat im WLTC einen Verbrauch von 5 l/100km [36], was einem mittleren Wirkungsgrad des Antriebsstranges von beachtlichen - 35\% entspricht. Dabei werden 132 gCO $/ \mathrm{km}_{\text {wLtc }}$ emittiert. Um 2021 den Grenzwert von 114 $\mathrm{gCO}_{2} / \mathrm{km}_{\text {WLTC }}$ (entspricht $95 \mathrm{gCO}_{2} / \mathrm{km}$ im NEDC ${ }^{4}$ ) zu erreichen, ist daher ein Wirkungsgrad von über $40 \%$ nötig. Um den Verbrauch um weitere 37,5 \% zu senken (Grenzwert 2030), wäre bereits ein Wirkungsgrad von $65 \%$ nötig - siehe auch Abbildung 17 (inklusive aller nötigen Randbedingungen). Das ist mehr als der theoretische maximal mögliche Wirkungsgrad einer VKM. Selbst bei einer Verringerung der nötigen Antriebsleitung (z.B. durch weiter verbesserte Aerodynamik) ist dieses Ziel unerreichbar.

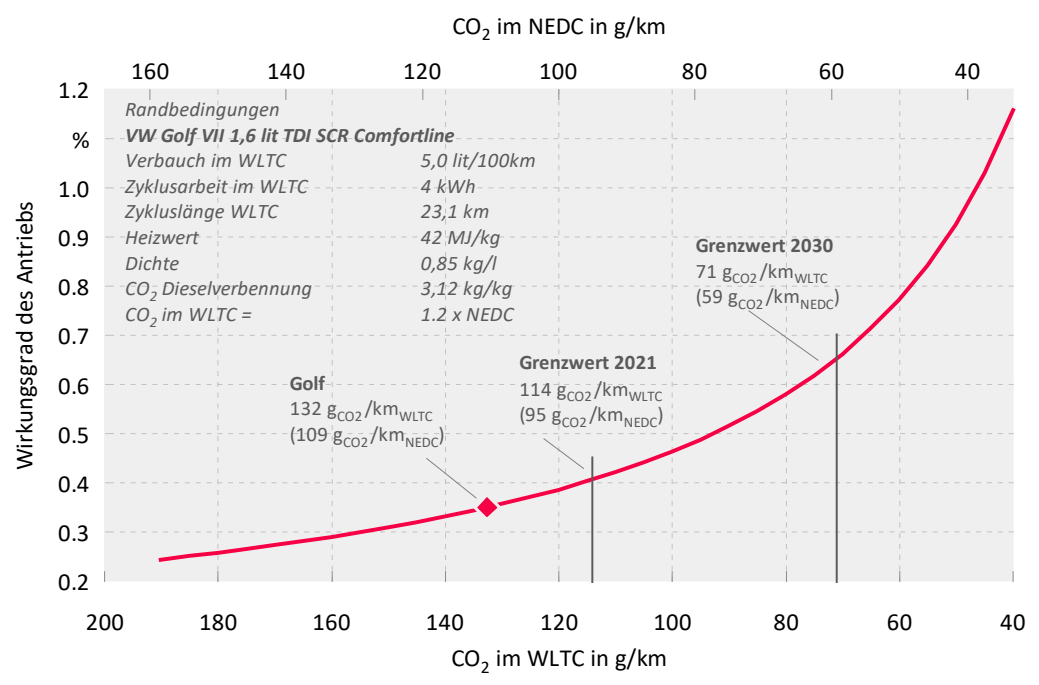

ABBILDUNG $17 \mathrm{CO}_{2}$ EMISSIONEN IM WLTC EINES GOLF VII DIESEL

Eine weitere Möglichkeit, die derzeit in Verbindung mit der Vernetzung der Fahrzeuge mit Informationstechnologien intensiv untersucht wird und die bei Nutzfahrzeugen bereits zu ersten Serienanwendungen geführt hat und die insbesondere für hybridisierte Pkw erfolgversprechend ist [37], ist die Nutzung GPS basierter Daten zur verbrauchsoptimierten Anpassung von Geschwindigkeitsverläufen an die Topologie, Geschwindigkeitsbeschränkungen etc. Dabei kann auch ein „Segeln“ in Schubphasen zielführend eingesetzt werden.

Bei Schienenfahrzeugen wurde in den letzten Jahren das Fahrverhalten im Hinblick auf eine Energieeinsparung in vielen Forschungsprojekten untersucht. Ergebnisse zeigen, dass die unterschiedliche Fahrweise von Fahrer/innen massive Auswirkungen auf den Energieverbrauch haben [38]. Eine effiziente Nutzung von Antriebsund Bremskräften ist für die Energiereduzierung wesentlich. Beispielsweise konnte auf einer $12 \mathrm{~km}$ langen Teststrecke durch die Reduktion des Bremsens von $18 \%$ auf $10 \%$ der Energieverbrauch um $38 \%$ reduziert werden [39]

Neben weniger Antriebs- und Bremsvorgängen kann auch durch niedrigere Maximalgeschwindigkeiten (im Beharrungsbereich) Energie eingespart werden, wenn das Ziel pünktlich erreicht werden kann [38]. Zudem kann durch die Nutzung der Auslaufphase durch antriebsloses Fahren (Ausrollen) Energie eingespart werden [40]. Es gibt daher viele Möglichkeiten durch unterschiedliches Fahrverhalten das Ziel pünktlich zu erreichen.

\footnotetext{
${ }^{4}$ zur Zeit wird von einem Umrechnungsfaktor zwischen NEDC und WLTC von 1,2 ausgegangen [52]
} 


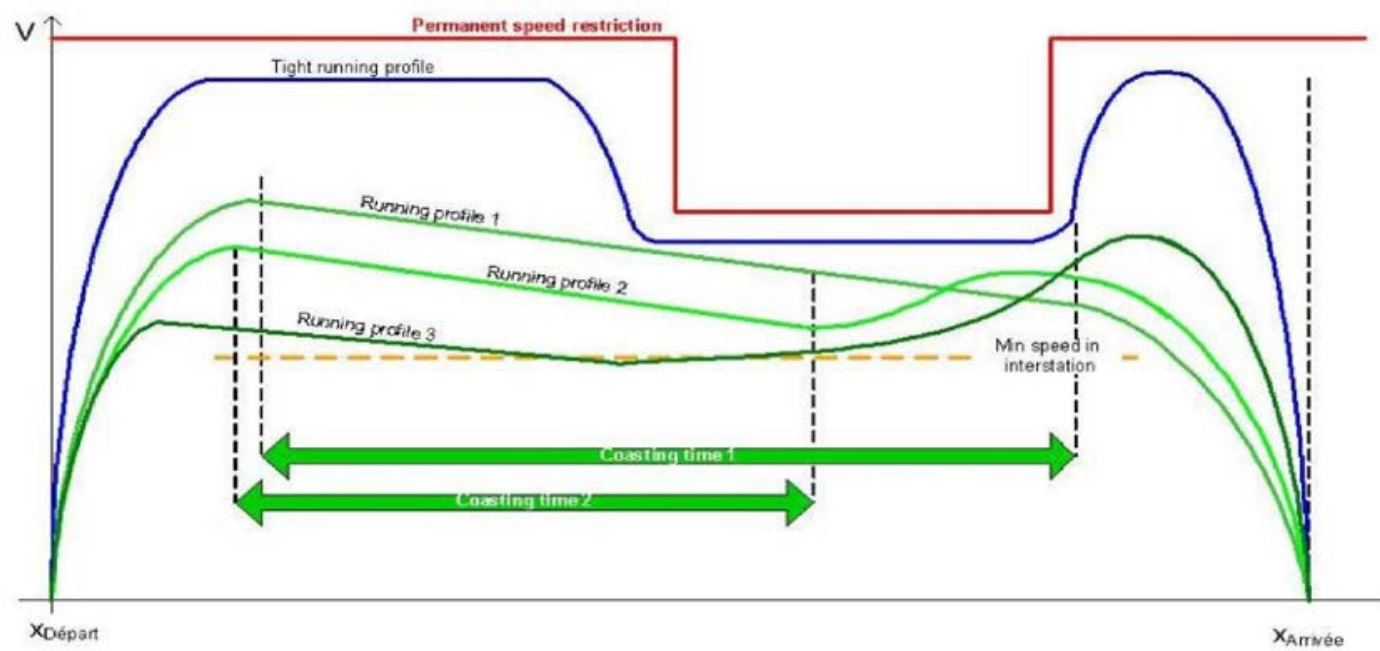

ABBILDUNG 18 ENERGIEEINSPARUNG DURCH UNTERSCHIEDLICHE FAHRPROFILE [40]

Großes Potential Energie einzusparen liegt daher in der Steuerung des Fahrverhaltens der Triebfahrzeugführer/innen bzw. Einhaltung eines optimierten Fahrprofils. Um dieses Potential zu heben, läuft derzeit das Projekt DISPO-SIM (laufendes FFG Projekt Uni-Salzburg, TUGraz). Dabei wird der Disponent unterstützt, um eine energieeffiziente Fahrweise bei Einhalten des Fahrplans zu erzielen. Das Programm zielt darauf ab, den Konflikt von Züge zu erkennen und netzweit eine optimierte Lösung zur Dispositionsentscheidung vorzuschlage. Dabei ist im Verspätungsfall die Minimierung der Verspätung das Ziel, im Regelfall die Minimierung des Energieverbrauchs.

Eine besondere und bezüglich $\mathrm{CO}_{2}$ Minderung verbrennungsmotorischer Antriebe sicher die aussichtsreichste Maßnahme besteht in der Anwendung alternativer Kraftstoffe. Die Gründe für das insgesamt herausragende Potenzial liegen in der hervorragenden Eignung von Verbrennungskraftmaschinen für unterschiedlichste flüssige und gasförmige Kraftstoffe, der extrem großen Verbreitung von VKMs sowie der bestehenden VerteilungsInfrastruktur. Der Einsatz alternativer flüssiger und gasförmiger Kraftstoffe wird allerdings wesentlich von der örtlichen und wirtschaftlich günstigen Verfügbarkeit abhängen - eine Konvergenz auf eine einzelne Lösung ist derzeit nicht absehbar.

Die Verbrennung im Motor stellt im Allgemeinen kein Problem dar. Manche alternative Kraftstoffe sind den fossilen Produkten so ähnlich, dass keinerlei Anpassung nötig ist. Weichen die Eigenschaften stärker ab (Dichte, Heizwert, Siedeverhalten, Sauerstoffgehalt etc.), müssen mitunter Veränderung am Motor vorgenommen werden. Manche Varianten versprechen - über den $\mathrm{CO}_{2}$-Vorteil hinaus - sogar wesentliches Potential zur Senkung der Schadstoffemissionen.

In den letzten Jahren hat sich die Diskussion über Kraftstoffe als Lösung für die $\mathrm{CO}_{2}$-Problematik intensiviert. Die prinzipielle Idee besteht darin, synthetisch erzeugte Kohlenwasserstoffe in - mehr oder weniger konventionellen Verbrennungsmotoren zu verbrennen. Bei Verwendung von erneuerbarer Energie als Ausgangsprodukt (Biomasse, grüner Strom) ergibt sich ein geschlossener $\mathrm{CO}_{2}-$ Kreislauf. Die Speicherung, die Verteilung und die Verwendung der Kraftstoffe basiert auf bewährten Technologien. Probleme mit der Verteilungsinfrastruktur und der Reichweite der Fahrzeuge entfallen. Als zusätzlicher Vorteil wird die Abfederung von temporärer Überproduktion von regenerativem Strom genannt.

Es gibt mehrere Prozesspfade, mit denen aus Strom unter Verwendung von $\mathrm{CO}_{2}$ und Wasserstoff flüssige, aber auch gasförmige, Kraftstoffe erzeugt werden können, z.B. über die Fischer-Tropsch Synthese (Diesel) oder über Methanisierung (Otto). Das Ausgangsprodukt $\mathrm{CO}_{2}$ wird durch eigene Prozesse generiert, kann aber auch direkt aus der Luft extrahiert werden. Der Wasserstoff wird durch Elektrolyse gewonnen. Kraftstoff der unter Verwendung von Strom als Energieträger verwendet wird, wird im Allgemeinen als „e-Fuel“ oder „PTx“ bezeichnet. 
Ein wesentlicher Nachteil der Methode ist allerdings der schlechte energetische Wirkungsgrad der Prozesse. Lediglich etwa 15\% der ursprünglich eingesetzten elektrischen Energie kann letztendlich in Form von Vortrieb umgesetzt werden, da bereits mit der Elektrolyse und folgender Synthese Wirkungsgradverluste einhergehen siehe Abbildung 19. Somit weist diese Variante einen wesentlich schlechteren Wirkungsgrad als die direkte Verwendung des Stroms in Elektrofahrzeugen auf (mit all ihren Nachteilen). Wollte man den gesamten Straßenverkehr auf e-Fuels umstellen, wäre eine Strommenge nötig, die einem Mehrfachen der gesamten heute produzierten Strommenge entspricht. Es stellt sich daher die Frage, in welcher Menge $\mathrm{CO}_{2}$-freier Strom lokal (in Europa) erzeugt werden kann. Wollte man den Strom zum Beispiel mittels Photovoltaik gewinnen, wäre das nur in Weltregionen mit hoher Sonneneinstrahlung sinnvoll möglich, womit das Problem der Abhängigkeit von Energieimporten bestehen bliebe.

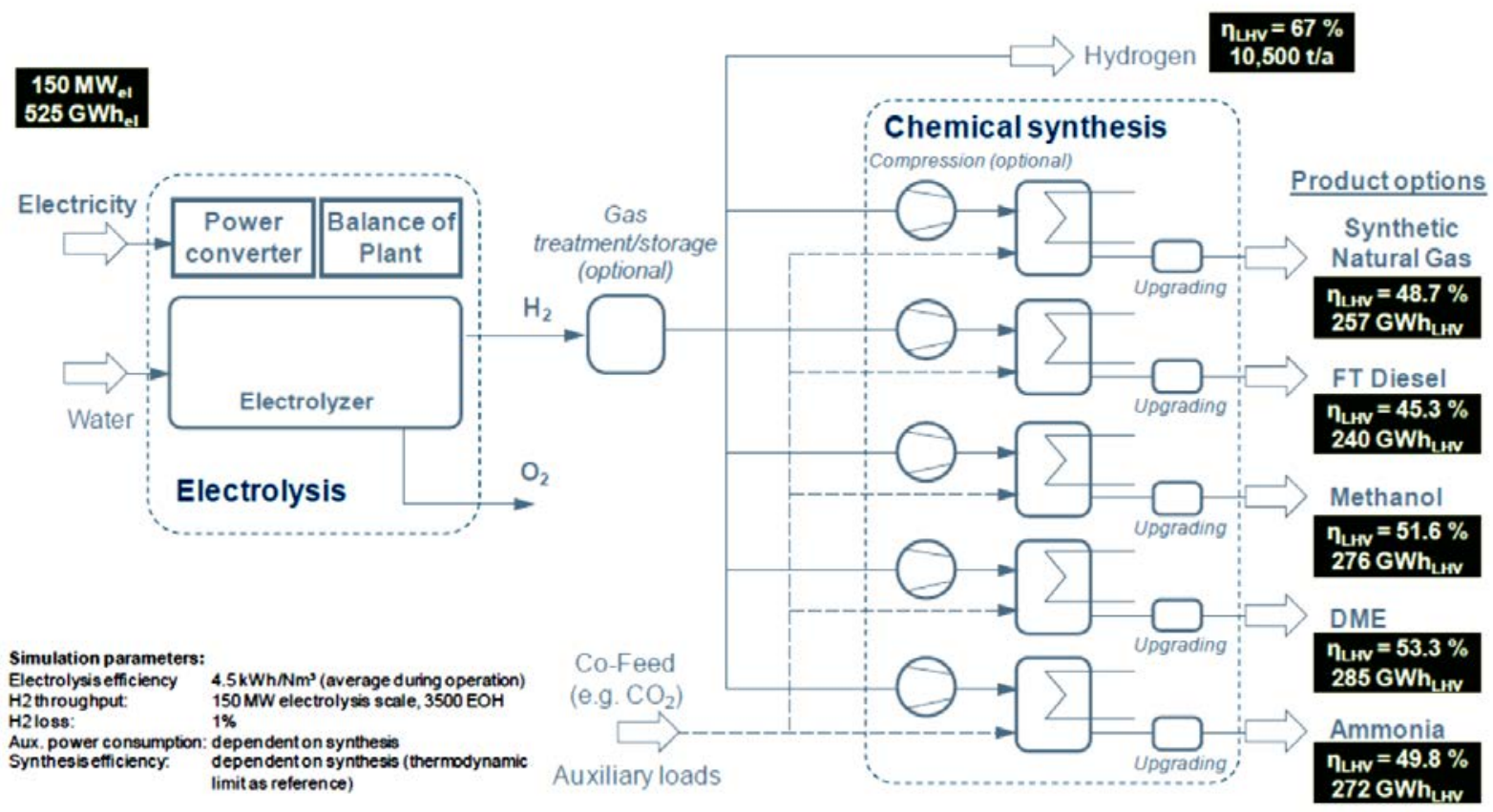

ABBILDUNG 19 WIRKUNGSGRADKETTE ALTERNATIVER KRAFSTOFFE WELL-TO-TANK [41]

Bei Hybrid-Antrieben und rein elektrischen Antrieben stellt die Speicherung der elektrischen Energie in der Batterie immer noch den eigentlichen Flaschenhals dar. Neben der Energie- und Leistungsdichte spielen insbesondere Fragen der Kosten, Lebensdauer, Temperaturabhängigkeit, Ladeverhalten sowie Sicherheit unter allen Bedingungen eine wesentliche Rolle. Für eine Kilowattstunde Speicherkapazität muss man aktuell (LithiumIonen Batterien) auf Pack-Ebene mit 170 - $200 €$ Kosten rechnen [42]. Will man beispielsweise eine reale Reichweite von $200 \mathrm{~km}$ gewährleisten, so benötigt man eine Batterie von ungefähr $40 \mathrm{kWh}$ [43] was derzeit einem Preis von ca. 7.500 Euro entspricht. Eine großflächige Umsetzung von PHEVs und BEVs scheint mittelfristig eher fraglich zu sein - unterschiedliche Schätzungen gehen von wenigen Prozent bis 18 \% im Jahr 2020 und 7 $30 \%$ im Jahr 2030 aus [44]. Generell wird erwartet, dass zukünftig unterschiedliche Antriebskonzepte parallel koexistieren und eine Spezialisierung auf die jeweiligen Anforderungen des Anwendungsbereiches stattfinden wird. So macht es durchaus Sinn, im urbanen Gebiet mit einer Flotte von Elektrofahrzeugen zu operieren (Paketzustelldienst, Taxi, Car-Sharing), wohingegen ein für die Allgemeinheit leistbares Familienauto mit dem Zweck, auch längere Distanzen zurückzulegen, mittelfristig (bis 2030) eher einen Verbrennungsmotor als Antrieb haben wird. Diese Einschätzung wird noch dadurch erhärtet, dass die Infrastruktur zur Treibstoffversorgung entlang des bestehenden Straßennetzes bestens vorhanden ist, aber für Elektrofahrzeuge - abgesehen von einzelnen Ladestationen - noch weitgehend fehlt und große flächendeckende Infrastrukturprojekte typischerweise Vorlaufzeiten von 20 - 30 Jahren benötigen.

Dies gilt natürlich auch für Wasserstoff, der in Verbindung mit Brennstoffzellen eine vielversprechende Variante der Elektromobilität bildet. Diese würden zum einen hohe Tank-to-Wheel Wirkungsgrade erlauben, da sie keine 
Wärmekraftmaschine und damit nicht mit dem Carnot-Wirkungsgrad limitiert sind [45], zum anderen eine Schadstoffemissionsfreiheit mit heute typischen Reichweiten von verbrennungsmotorischen Antrieben kombinieren. Die mittleren Tank-to-Wheel Wirkungsgrade für Pkw liegen derzeit etwa bei $50-55 \%$. Neben der Wasserstoff-Infrastrukturfrage verbleiben hier die derzeit hohen Kosten eine Hürde, an deren Überwindung geforscht wird.

Zum Thema Wasserstoff ist anzumerken, dass die Zillertaler-Bahn als erste Bahn plant ihren Betrieb zur Gänze auf Wasserstoffantrieb umzustellen. Zudem wird aktuell das FFG Projekt HYTRAIL (HyCentA Research GmbH, Montanuniversität Leoben - Lehrstuhl für Energieverbundtechnik, Energieinstitut an der Johannes Kepler Universität Linz, synergesis consult.ing, Technische Universität Graz - Institut für Eisenbahnwesen und Verkehrswirtschaft), bearbeitet, das die infrastrukturseitigen Randbedingungen zur Anwendung von Wasserstoffantrieben darlegt.

Vor allem für den schadstofffreien LKW-Fernverkehr sind Brennstoffzellen-Antriebe eine reizvolle und vermutlich als erstes in die breite Anwendung gehende Alternative, da dort die absehbaren Reichweiten und Ladezeiten batterieelektrischer Antriebe nicht ausreichend sind.

Etwas unterschiedlich sieht die Situation bei Zweiradfahrzeugen aus: Diese werden zukünftig nicht nur hinsichtlich Schadstoffemission, sondern in Bezug auf treibhausgasrelevante Emissionen und Energieeffizienz weltweit einen deutlich höheren Stellenwert bekommen, da bei dieser Fahrzeuggruppe heute der Wirkungsgrad sehr deutlich hinter jenem von Pkw zurückbleibt und bis 2020 mit über 100 Mio. motorisierten Zweirädern jährlich von einer stückzahlbezogen gleich hohen Neuproduktion wie bei Pkw auszugehen ist. Damit entsteht in Verbindung mit dem derzeit sehr ungünstigen Wirkungsgrad der Einfach-CVT Getriebe, die bei Mopeds und Kleinmotorrädern einen ganz wesentlichen Anteil der Flotte ausmachen, ein der Transportleistung unangemessen hoher $\mathrm{CO}_{2}$-Anteil.

In Verbindung mit den hohen Schadstoffemissionen der heutigen, teilweise veralteten Motorentechnologie asiatischer Low-Cost Zwei- und Dreiradfahrzeuge ist der vorgeschriebene Einsatz von E-Bikes in Ballungszentren durchaus verständlich. So ist der abgeschätzte Gesamtmarkt für E-Bikes 2018 weltweit ca. 42 Mio., dominiert mit über 90 \% von Asien bzw. China. Bemerkenswert zu sehen ist, dass die in China durchschnittlich nur 167 USD kostenden Low-Cost Fahrzeuge Bleibatterietechnik verwenden, der Anteil von Li-lon Batterien ist nur $4 \%$ [46]. Technologisch können diese damit nicht mit den etwa 1,5 Mio in Europa verkauften, vorwiegend als Pedelecs ausgeführte E-Bikes verglichen werden, die mit durchschnittlich 1550 US Dollar (65 \% Li-lon Anteil) etwa den Faktor 10 mehr kosten. Durch mangelhaftes Recycling führen die Bleibatterien in China zudem zu beträchtlichen Umweltproblemen. Sehr vorteilhaft sind die $\mathrm{CO}_{2}$ Emissionen der E-Bikes, welche wesentlich geringer als bei Pkw liegen, weshalb diese - unter der Voraussetzung moderner Speichertechnik- durchaus eine Alternative zum Pkw darstellen könnten.

\section{ZUSAMMENFASSUNG UND AUSBLICK}

Bei den „Verkehrsmitteln“ werden aus Umweltsicht zwei Punkte als besonders relevante Herausforderungen gesehen. Dies sind die Schadstoffemissionen und der Einsatz von Primärenergie und wertvollen Rohstoffen über den gesamten Lebenszyklus des Verkehrsmittels mit der damit verbundenen Treibhausgasrelevanz, insbesondere dem $\mathrm{CO}_{2}$ Ausstoß.

Hinsichtlich der Schadstoffe stellen die Partikel(anzahl)-Emission und insbesondere Stickoxide (und hier vor allem die $\mathrm{NO}_{2}$ Emission), für die der Straßenverkehr insbesondere durch Dieselmotoren einer der Hauptverursacher ist, die besondere Herausforderung dar. Der Verbesserungsbedarf wurde durch die Anwendung realitätsnäherer Testprozeduren (Stichwort Real Drive Emission RDE) aufgezeigt, die in Europa beim LKW bereits in Kraft sind und seit 2018 beim Pkw zur Anwendung kommen. Dies wird real zu einer wesentlichen Verbesserung in emissionsbelasteten Gebieten führen, allerdings ist mit der derzeitigen Zusammensetzung der Fahrzeugflotte und deren Erneuerungszyklus mit einer zeitlichen „Verschleifung“ zu rechnen, die beispielsweise durch lokale 
Einschränkungen etwas beschleunigt werden kann. Ebenso sind bei den zweifellos noch erforderlichen Maßnahmen zur Senkung der Schadstoffemissionen bei Verkehrsmitteln stationäre Quellen (Feuerungsanlagen, Verbrennungsmotoren, Industrie, Landwirtschaft etc.) bei den Maßnahmen entsprechend ihrem Beitrag zu berücksichtigen, um einen effizienten Einsatz der erforderlichen Ressourcen sicherzustellen und damit eine Minderung der Immissionswerte auf einem Niveau unterhalb der gesundheitlichen Relevanz zu erreichen.

Vor dem Hintergrund der Funktionseigenschaften und des erreichten Reifegrades, der bestehenden Infrastruktur und der wirtschaftlichen Rahmenbedingungen ist aus Sicht der Autoren auf absehbare Zeit (>20 Jahre) von der Dominanz verbrennungsmotorischer Antriebe mit Hybridisierung in Pkw und LKW auszugehen. Diese verfügen trotz einer langen Entwicklungsgeschichte über hohes Potenzial zur Weiterentwicklung, welches hinsichtlich Schadstoffemissionen bei entsprechendem Aufwand auf ein Niveau gebracht werden kann, welches deutlich mehr als eine Größenordnung unter den sonstigen Emittenten (Landwirtshaft, Hausbrand, Industrie) liegt (,zero impact").

Bezüglich der Emissionen treibhausgasrelevanter Komponenten können Verbesserungen durch eine Steigerung des Wirkungsgrades mittels der angesprochenen motorseitigen Maßnahmen sowie einer Teilelektrifizierung erreicht werden. Ein wesentlicher Hebel ist durch die Zusammensetzung der Fahrzeugflotte (lenkender Einfluss durch steuerliche Rahmenbedingungen, wie in manchen Ländern bereits ausgeprägt in Kraft), die Kraftstoffpreisgestaltung sowie die Einbeziehung des Produktionsprozesses in die Bewertung gegeben. Die wesentlichste Einflussmöglichkeit besteht jedoch im Einsatz alternativer flüssiger und gasförmiger [47] Kraftstoffe. Dies gilt natürlich zunächst unabhängig vom Energiewandler im Fahrzeug. Im Fall des verbrennungsmotorischen Antriebes besteht eine Vielzahl von Möglichkeiten wie beispielsweise Power-to-Gas, Zumischung regenerativ hergestellten Wasserstoffes in das bestehende Erdgasnetz und Nutzung im Fahrzeug [48], Methanisierung von Wasserstoff [49], Erzeugung (synthetischer) Kraftstoffe auf Basis nachwachsender Rohstoffe [50] und von Wasserstoff, um nur einige zu nennen, scheinen mit vertretbarem Aufwand darstellbar. Ein wesentlicher Vorteil ist deren Rückwärtskompatibilität sowie die vorhandene Infrastruktur.

Neben der Teilelektrifizierung sind rein batterieelektrische Antrieben eine Möglichkeit der lokal schadstofffreien Energiewandlung. Deren Vorteile können insbesondere für urbane Mobilität und Nahverkehr genutzt werden. Die Ladeinfrastruktur und die Speicherung der elektrischen Energie in der Batterie stellt für Langstreckenverkehr immer noch den eigentlichen Flaschenhals dar. Neben der Energie- und Leistungsdichte spielen insbesondere Fragen der Kosten, Lebensdauer, Temperaturabhängigkeit, Ladeverhalten sowie Sicherheit unter allen Bedingungen eine wesentliche Rolle. Bei der Bewertung der $\mathrm{CO}_{2}$-Bilanz von elektrischen Antrieben ist es wichtig, eine Gesamtsystembetrachtung vorzunehmen: So ist der gesamte Lebenszyklus von der Erzeugung des Verkehrsmittels über den Betrieb bis zur Entsorgung incl. Recycling sowie die Errichtung der Infrastruktur ebenso wie der Vorgang der Stromerzeugung zu berücksichtigen. Diese erfolgt teilweise mit sehr niedrigen Wirkungsgraden und auf Basis von Kohle, was zu deutlich höherer Treibhausrelevanz als ein verbrennungsmotorischer Antrieb führt. Als eine klare Fehlbewertung wird aus Sicht der Autoren daher die Berücksichtigung von elektrischer Energie als $\mathrm{CO}_{2}$-frei in den EU Gesetzen gesehen, die naturgemäß eine entsprechende Entwicklung der Fahrzeugtechnik u.a. mit sehr leistungsstarken und großen Plug-In Fahrzeugen zur Folge hat; für deren reale Bilanz wird auch das Nutzerverhalten (Langstreckenfahrten oder häufiger Betrieb ohne Aufladen) eine entscheidende Rolle spielen.

Eine deutlich bessere Situation besteht bei kleinen Zweirädern, die bezüglich Reichweite weniger anspruchsvoll sind. Diese weisen als E-Bikes nicht nur weniger Ressourcenbedarf, sondern auch deutlich höhere Wirkungsgrade als verbrennungsmotorische Antriebe auf und sind zudem lokal schadstofffrei.

Eine Bewertung verschiedener $\mathrm{CO}_{2}$-Minderungskonzepte für Pkw Antriebe ist in der folgenden Tabelle zusammengefasst. Bei der Bewertung der elektrischen Energie wird dabei von einer Bandbreite zukünftiger, vorwiegend regenerativ erzeugter Energie ausgegangen. Die Tabelle gibt die Sicht der Autoren wieder und stellt den Versuch einer zusammenfassenden Quantifizierung dar. Die Werte basieren auf unterschiedlichsten internen und externen Quellen und Erfahrungswerten. 


\begin{tabular}{|c|c|c|c|c|c|c|c|c|}
\hline $\begin{array}{l}\text { Kundenwerte } \\
\text { Eigenschaften }\end{array}$ & Basis: Otto & Diesel & $\begin{array}{l}\text { Erdgas } \\
\text { monovalent }\end{array}$ & $\begin{array}{l}48 \mathrm{~V} \\
\text { Elektrifiziert }\end{array}$ & $\begin{array}{l}\text { HEV - } \\
\text { Otto }\end{array}$ & $\begin{array}{l}\text { PHEV - } \\
\text { Otto }\end{array}$ & $E V$ & $\begin{array}{l}\mathrm{H} 2 \\
\text { Brennstoff- } \\
\text { zelle }\end{array}$ \\
\hline $\begin{array}{l}\mathrm{CO}_{2} \text { Vermeidung } \\
\text { im NEDC }\end{array}$ & $\begin{array}{l}0 \mathrm{~km} \\
\text { (Basis) }\end{array}$ & $20 \%$ & $25 \%$ & $5 \%$ & $18 \%$ & $36-60 \%$ & $55-100 \%$ & $55-100 \%$ \\
\hline $\begin{array}{l}\text { Lokale } \\
\text { Emissionsfreiheit } \\
\text { (elektrische } \\
\text { Fahranteile) }\end{array}$ & $0 \mathrm{~km}$ & $0 \mathrm{~km}$ & $0 \mathrm{~km}$ & $0 \mathrm{~km}$ & $1-5 \mathrm{~km}$ & $16-50 \mathrm{~km}$ & $>150 \mathrm{~km}$ & $>600 \mathrm{~km}$ \\
\hline Relative Kosten & 1 & 1,3 & $1,1-1,2$ & 1,2 & Ca. 2-3 & Ca. $3-3,5$ & Ca. 3 & $?$ \\
\hline Gesamtreichweite & $>600 \mathrm{~km}$ & $>800 \mathrm{~km}$ & $>500 \mathrm{~km}$ & $>600 \mathrm{~km}$ & $>600 \mathrm{~km}$ & $>600 \mathrm{~km}$ & $>150 \mathrm{~km}$ & $>600 \mathrm{~km}$ \\
\hline
\end{tabular}

\section{TABELLE 1: BEWERTUNG VERSCHIEDENER $\mathrm{CO}_{2}$-MINDERUNGSKONZEPTE FÜR PKW ANTRIEBE.}

Im Straßen-Güterverkehr wird wegen der deutlich höheren Anforderungen hinsichtlich Reichweite und Lastkollektive ein batterieelektrischer Antrieb - abgesehen von Kommunalfahrzeugen und dem innerstädtischen Güterverkehr - in absehbarer Zeit keine nennenswerte Rolle spielen. Der verbrennungsmotorische Antrieb wird über längere Zeit dominant bleiben, vorteilhafterweise mit $\mathrm{CO}_{2}$-freien Kraftstoffen. Eine mittel- und langfristig aussichtsreiche Alternative ist ein Antrieb auf Basis einer $\mathrm{H}_{2}$-Brennstoffzelle.

Ganz anders ist die Situation beim Personentransport im urbanen Bereich, hier spielt die Elektrotraktion ab einer Einwohnerzahl von etwa 100000 mit der Straßenbahn sowie der U-Bahn ab etwa 500000 Einwohnern bereits heute eine wesentliche bzw. die dominante Rolle. Mit der ausgeprägten Zunahme von großen Ballungsräumen und Megacitys wird dieser Trend zukünftig noch deutlich verstärkt.

Wegen der auch zukünftig noch absehbaren Dominanz verbrennungsmotorischer Antriebe für Fahrzeuge (siehe Argumentation von zuvor) ist die derzeitige Fokussierung der Forschungsarbeiten alleine auf den batterieelektrischen Antrieb zu überdenken. Mit einem wesentlichen Teil der Ressourcen sollten die anderen oben genannten Alternativen intensiver untersucht werden.

\section{LITERATUR}

[1] H. Schmidt und G. Zielonka, „Worldwide Harmonised Light-duty vehicles Test Procedure - Gesetzgebung und praktische Umsetzung," in 39. Wiener Motorensymposium, Wien, 2018.

[2] L. Ntziachristos, S. Geivanidis, Z. Samaras, A. Xanthopoulos, H. Steven und B. Bugsel, „Study on possible new measures concerning motorcycle emissions," Report No: 08.RE.0019.V4 for European Commission DG Enterprise and Industry - Automotive Unit Avenue d'Auderghem; 45 B-1040 Brussels; Scientific Responsible Prof. Zissis Samaras; Thessaloniki, 2009.

[3] S. Hausberger, M. Rexeis, R. Luz und N. Furian, „Die Rolle von CO2 und RDE in der zukünftigen Emissionsgesetzgebung von PKW und Nutzfahrzeugen," in 14. Tagung "Der Arbeitsprozess des Verbrennungsmotors", Graz, 2013.

[4] R. Ratzberger, Investigation of Robust Close-Coupled Diesel Exhaust Aftertreatment for Passenger Cars with 12 V and 48 V Architecture, Graz: Dissertation, Technische Universität Graz, 2018.

[5] S. Hausberger und M. Schwingshackl, Verkehrsszenarien für WAMplus im Rahmen des MonMech 2013, Institut für Verbrennungskraftmaschinen und Thermodynamik; TU Graz; 2013.

[6] R. Kurz, C. Orthofer, P. Sturm, A. Kreiser, U. Uhrner, R. Reifeltshammer und M. Rexeis, „Projection of the Air Quality in Vienna between 2005 and 2020 for NOx and PM10,“ Urban Climate 10, pp. 709-719, 2014. 
[7] M. Krüger, J. Hammer, A. Kufferath, R. Maier und D. Naber, „Verbrauch im Einklang mit Realemissionen; die Zukunft für den Diesel Pkw,“ in 38. Internationales Wiener Motorensymposium, Wien, 2017.

[8] M. Goergen, M. Nijs, H. Lehn, J. Scharf, M. Thewes, M. Hendrikx, J. Claßen, S. Sterlepper und H. Baumgarten, „Current and Future Trends of Gasoline Particulate Filter Technologies, Calibration Strategies and Aging Methods, “ in Aachen Colloquium 2017, Aachen, 2017.

[9] E. Jean, „GPF, Just a DPF for Gasoline Engines?,“ in Aachen Colloquium, Aachen, 2017.

[10] A. Kufferath, M. Krüger, D. Naber, E. Mailänder und R. Maier, „Der Diesel Powertrain auf dem Weg zu einem vernachlässigbaren Beitrag bei den NO2-Immissionen in den Städten,“ in 39. Internationales Wiener Motorensymposium, Wien, 2018.

[11] N.N., „Moderne Diesel mit Euro 6d-TEMP sind sehr sauber | ADAC,“ ADAC e.V., 18 September 2018. [Online]. Available: https://www.adac.de/rund-ums-fahrzeug/abgas-diesel-fahrverbote/dieselkaufabgasnorm/euro-6d-temp/. [Zugriff am 2501 2019].

[12] ADAC, „ADAC EcoTest - Fünf Sterne für die Umwelt,“ [Online]. Available: https://www.adac.de/infotestrat/tests/eco-test/default.aspx. [Zugriff am 2002 2019].

[13] D. Köhler, „NOx und Feinstaub - Grenzwerte bei Lungenärzten umstritten : www.lungenaerzte-imnetz.de, " 2301 2019. [Online]. Available: NOx und Feinstaub - Grenzwerte bei Lungenärzten umstritten : www.lungenaerzte-im-netz.de. [Zugriff am 2501 2019].

[14] M. Koers, „ $\mathrm{CO}_{2}$-Regulierung bei PKW und leichten Nutzfahrzeugen - VDA,“ [Online]. Available: https://www.vda.de/de/themen/umwelt-und-klima/co2-regulierung-bei-pkw-und-leichten-nfz/co2regulierung-bei-pkw-und-leichten-nutzfahrzeugen.html. [Zugriff am 31 Januar 2019].

[15] N.N., „Autoindustrie entsetzt über schaerfere CO2-Grenzwerte in der EU,“ Tiroler Tagseszeitung Onlineausgabe, $\quad 18 \quad 12 \quad 2018 . \quad$ [Online]. Available: https://www.tt.com/politik/europapolitik/15138840/autoindustrie-entsetzt-ueber-schaerfere-co2grenzwerte-in-der-eu. [Zugriff am 2501 2019].

[16] L 349, Amtsblatt der Europäischen Union, 2017.

[17] Handelsblatt GmbH, „Autokonzerne wollen mit Sprit aus Strom CO2 einsparen,“ 1 Oktober 2018. [Online]. Available: https://www.handelsblatt.com/politik/deutschland/e-fuels-autokonzerne-wollenmit-sprit-aus-strom-co2-einsparen-die-regierung-laesst-sie-aber-nicht/23134986.html?ticket=ST1931428-ytllyfsfMqZgSMa5UcwN-ap3. [Zugriff am 2002 2019].

[18] N.N., „Kompaktversion - ÖAMTC Expertenbericht Mobilität \& Klimaschutz 2030,“ Österreichischer Automobil-, Motorrad- und Touringclub (ÖAMTC), Wien, 2018.

[19] T. Pauer, H. Yilmaz, J. Zumbrägel, W. Wiese, P. Rogler und E. Schünemann, „Die neue Generation Benzin Direkteinspritzsysteme von Bosch,“ in 38. Internationales Wiener Motorensymposium, Wien, 2017.

[20] C. Severin, F. Bunar, M. Brauer, M. Diezemann, W. Schultalbers, G. Buschmann, M. Kratzsch und K. Blumenröder, „Potentiale einer hochintegrierten Abgasnachbehandlung für zukünftige PKW Dieselmotoren, “ in 38. Internationales Wiener Motorensymposium, Wien, 2017. 
[21] J. Grimm, G. Avolio, O. Maiwald, R. Brück und G. Rösel, „Energiebasierte Optimierung eines Dieselhybrids für die zukünftige „Real Driving“ Emissionsgesetzgebung,“ in 38. Internationales Wiener Motorensymposium, Wien, 2017.

[22] J. Scharf, J. Ogrzewalle, T. Uhlmann, M. Thewes, A. Balazs, P. Grzeschik, S. Sonnen, H. Baumgartner, A. Nase, M. Witler und D. Heckmann, „Ottomotoren für Hybridantriebe - Hochtechnologie oder Low-Cost Aggregate?,“ in 38. Internationales Wiener Motorensymposium, Wien, 2017.

[23] C. Ernst, O. I, N. Neumann, C. Harter und L. Eckstein, „Supplier Strategies 2025 - Winning Technologies for the CO2 Challenge," in 24th Aachener Colloquium Automobile and Engine Technology, Aachen, 2015.

[24] N.N., „ÖAMTC Expertenbericht Mobilität \& Klimaschutz 2030,“ Österreichischer Automobil-, Motorradund Touringclub (ÖAMTC), Wien, 2018.

[25] Handelsblatt $\mathrm{GmbH}$, „CO2-Bilanzen: Das goldene Kalb ist schlachtreif,“ 8 November 2012. [Online]. Available: $\quad$ https://www.handelsblatt.com/auto/nachrichten/co2-bilanzen-das-goldene-kalb-istschlachtreif/7358112-all.html?ticket=ST-1450946-WCVGXtGTT6dKaAyMqPVo-ap3. [Zugriff am 1 Februar 2019].

[26] Eurostat, „Electricity generation statistics - first results - Statistics Explained,“ April 2018. [Online]. Available: https://ec.europa.eu/eurostat/statisticsexplained/index.php?title=Electricity_generation_statistics_\%E2\%80\%93_first_results\#Production_of_ electricity. [Zugriff am 1 Februar 2019].

[27] The World Bank, „World Development Indicators | The World Bank,“ 2017. [Online]. Available: http://wdi.worldbank.org/table/3.7. [Zugriff am 01 Februar 2019].

[28] M. Beermann, L. Canella und G. Jungmeier, „Treibausgasemissionen der Stromerzeugung und Transportdienstleistung von E-Fahrzeugen in Österreich," in Symposium Energieinnovation, Graz, 2012.

[29] E. Schutting, J. Ratzinger und H. Eichlseder, „Well-to-Wheel A Comparison of Propulsion Systems, “ in 11th A3PS Conference ECO-MOBILITY 2016, Wien, 2016.

[30] C. M. Schwarzer, „Energiebilanz: So sauber ist das Elektroauto | ZEIT ONLINE,“ 16 Januar 2014. [Online]. Available: https://www.zeit.de/mobilitaet/2014-01/elektroauto-energiebilanz. [Zugriff am 01 Februar 2019].

[31] L. Möhring und J. Andersen, „Erdgasmobilität - skalierbarer und bezahlbarer Umwelt- und Klimaschutz jetzt, “ in 38. Internationales Wiener Motorensymposium, Wien, 2017.

[32] L. Leiva, „Wie stark belastet die Batterieherstellung die Ökobilanz von Elektroautos? - Energie Experten,“ 7 Dezember 2017. [Online]. Available: https://www.energie-experten.ch/de/mobilitaet/detail/wiestark-belastet-die-batterieherstellung-die-oekobilanz-von-elektroautos.html. [Zugriff am 23 April 2019].

[33] W. Buchholz, J. Frank, H. Karl, J. Pfeiffer, K. Pittel, U. Triebswetter, J. Habermann, W. Mauch und T. Staudacher, „Die Zukunft der Energiemärkte,“ ifo Institut, München, 2012.

[34] H. Helms, J. Jöhrens, C. Kämper, J. Giegrich, A. Liebich, R. Vogt und U. Lambrecht, „Weiterentwicklung und vertiefte Analyse der Umweltbilanz von Elektrofahrzeugen UBA-FB 002316, “ Umweltforschungsplan des Bundesministeriums für Umwelt, Naturschutz, Bau und Reaktorsicherheit, Dessau-Roßlau, 2016. 
[35] S. Rommel, R. Geiger, R. Schneider, M. Baumann, P. Brandstetter, M. Held, S. Albrecht, O. Creutzenberg und C. Dasenbrock, „Leichtbau in Mobilität und FertigungÖkologische Aspekte," e-mobil BW GmbH Landesagentur für Elektromobilität und Brennstoffzellentechnologie, Stuttgart, 2012.

[36] ADAC Versicherung AG, „VW Golf 1.6 TDI SCR Comfortline ADAC Info - Autodatenbank Detailseite,“ [Online]. Available: https://www.adac.de/infotestrat/autodatenbank/autokatalog/detail.aspx?mid=294842\&bezeichnung= vw-golf-1-6-tdi-scr-comfortline. [Zugriff am 2602 2019].

[37] M. Kluin, H. Maschmeyer, S. Jenkins und C. Beidl, „Simulations- und Testmethoden für Hybridfahrzeuge mit vorausschauendem Energiemanagement," in 5.Internationales Symposium für Entwicklungsmethodik, Wiesbaden, 2013.

[38] B. Bienfait, P. Zoetardt und B. Barnard, „AUTOMATIC TRAIN OPERATION: THE MANDATORY IMPROVEMENT FOR ETCS APPLICATIONS,“ in ASPECT 2012, London, 2012.

[39] M. Marinov, „Sustainable Rail Transport,“ in RailNewcastle Talks, Newcastle, 2016.

[40] C. Gralla, „Automatisches Fahren als Antwort auf Digitalisierung 4.0?,“ in 43. Tagung Moderne Schienenfahrzeuge, Graz, 2016.

[41] A. Tremel, „Green hydrogen and downstream Green hydrogen and downstream fuels for the transportation sector, " in 4. Internationaler Motorenkongress, Baden Baden, 2017.

[42] M. Heerwagen, „How CO2 is Transforming the Automotive Industry,“ ATZ worldwide, pp. 8-13, Februar 2019.

[43] A. Proissl, „E-Auto Verbrauchstest: Stromsparer und Stromfresser | trend.at,“ 10 2018. [Online]. Available: https://www.trend.at/branchen/auto-mobilitaet/e-auto-verbrauchstest-stromsparerstromfresser-10428721. [Zugriff am 2205 2019].

[44] Frauenhofer Institut für Systeme und Innovationsforschung, „Produkt-Roadmap Lithium-Ionen-Batterien 2030," Februar 2012. [Online]. Available: https://www.isi.fraunhofer.de/content/dam/isi/dokumente/cct/lib/PRM-LIB2030.pdf. [Zugriff am 01 Februar 2019].

[45] H. Eichlseder und M. Klell, Wasserstoff in der Fahrzeugtechnik, pringer Vieweg Verlag, 2012.

[46] J. Shuguang, R. C. Christopher, J. B. Matthew, Y. Wu und D. M. Julian, „Electric vehicles in China : Emissions and health impacts," Environmental Science and Technology, pp. 2018-2024, 2012.

[47] H. Eichlseder, "Gasmotorenkonzepte für mobile Anwendungen," in Tagung Motorische Verbrennung, Stuttgart, 2013.

[48] R. Otten, „Das Audi E-Gas Project - Ganzheitliche Bilanzierung als Treiber für einen neuen Blick auf die Automobile Zukunft,“ in 23. AVL Konferenz „Motor und Umwelt", Graz, 2012.

[49] H. Eichlseder, K. Schaffer, D. Leitner, M. Klell und M. Sartory, Potential of Synergies in a Vehicle for Variable Mixtures of CNG and Hydrogen, SAE Technical Paper 2009-01-1420, 2009. 
[50] G. Merker und R. Teichmann, Grundlagen Verbrennungsmotoren, Springer Vieweg, 2014.

[51] B. Berman, „What the Tesla Model S Battery Replacement Price Doesn't Say | PluginCars.com,“ 04 Dezember 2012. [Online]. Available: https://www.plugincars.com/tesla-model-s-replacement-batterypacks-125571.html. [Zugriff am 01 Februar 2019].

[52] Institut der deutschen Wirtschaft IWD, „Klimapolitik: Die CO2-Welt - iwd.de, “ 9 November 2017. [Online]. Available: https://www.iwd.de/artikel/klimapolitik-die-co2-welt-366033/. [Zugriff am 2002 2019].

[53] United States Environmental Protection Agency, „Global Greenhouse Gas Emissions Data, “ [Online]. Available: https://www.epa.gov/ghgemissions/global-greenhouse-gas-emissions-data. [Zugriff am 2002 2019].

[54] J. G. Olivier, G. Janssens-Maenhout, M. Muntean und J. A. Peters, „TRENDS IN GLOBAL CO2 EMISSIONS,“ PBL Netherlands Environmental Assessment Agency, The Hague, 2016.

[55] Umweltbundesasmt Deutschland, „Daten zum Verkehr,“ Umweltbundesamt, Dessau, 2012.

[56] European Environment Agency, „How are emissions of greenhouse gases by the EU evolving?,“ [Online]. Available: https://ec.europa.eu/eurostat/cache/infographs/energy-2017/bloc-4a.html. [Zugriff am 20 02 2019].

[57] Europäische Komission, EU Transport in Figures, Europäische Union, 2018.

[58] U. Winzen, „WLTP treibt CO2-Wert,“ Automobilwoche, p. 18, 18 Februar 2019.

[59] ADAC, „ADAC EcoTest - Fünf Sterne für die Umwelt,“ 19 März 2019. [Online]. Available: https://www.adac.de/_mmm/pdf/27473_118924.pdf. [Zugriff am 23 April 2019].

[60] ADAC, „ADAC EcoTest - Fünf Sterne für die Umwelt,“ 19 März 2019. [Online]. Available: https://www.adac.de/_mmm/pdf/FTKInfo\%20EcoTest\%20Test\%20und\%20Bewertungskriterien\%20ab\%2012_2016_936KB_292234.pdf. [Zugriff am 23 April 2019]. 